\title{
Finding eigenvalues and eigenfunctions of the Zaremba problem for the circle
}

\author{
Ari Laptev, Anastasiya Peicheva and Alexander Shlapunov
}

\begin{abstract}
We consider Zaremba type boundary value problem for the Laplace operator in the unit circle on the complex plane. Using the theorem on the exponential representation for solutions to equations with constant coefficients we indicate a way to find eigenvalues of the problem and to construct its eigenfunctions.
\end{abstract}

Mathematics Subject Classification (2010). 47A10; 35J57; 30B60.

Keywords. Sturm-Liouville problems, Robin condition, eigenvalues.

\section{Introduction}

The spectral theory gives powerful tools to investigate operator equations (see, for instance, [10], [7], [9]). The classical Hilbert space approach helps to handle both selfadjoint and non-selfadjoint problems in (weighted) Sobolev type spaces over different types of domains (smooth domains, Lipschitz domains, domains with conical and edges singularities etc.), see, for instance, $[5],[6],[2],[12],[3],[4],[8],[22]$ and many others. Recently the approach was adopted to a wide class of non-coercive mixed boundary problems, see [19], [20].

Apart from the theory, the problem is to find the corresponding eigenvalues and to construct the eigenfunctions. The use of the Fourier method of separation of variables have lead to nice examples in classical PDE's (see for example [23, Suppl. II, P. 1, §2] for the coercive or [19] for non-coercive problems). However it does not work in many cases even for mixed problems for the Laplace equation, cf. the example for the Zaremba problem (i.e. a Robin type boundary problem with the Dirichlet condition on a part $S$ of the boundary $\partial D$ of the domain $D$ in the Euclidean space and the Neumann condition on the complement of $S$ on $\partial D$, see [25]).

In the present paper, using the theorem on the exponential representation for solutions to equations with constant coefficients (see [15, Introduction, p. 19]), we indicate a way to present the eigenfunctions of the (generally, 
non-coercive) Zaremba type problem via an (infinite) sums of the Bessel functions in the unit circle on the complex plane. On this way we produce also a criterion for identifying the corresponding eigenvalues. We emphasize that unlike to the Dirichlet problem (or to the Neumann problem) it is impossible to compute corresponding eigenvectors as finite sums of the Bessel functions.

\section{The Zaremba type problem for the unit disk}

Let $\mathbb{R}^{2}$ be the real plane with the coordinates $x=\left(x_{1}, x_{2}\right)$ and $\mathbb{C}$ be the complex plane with the complex structure given by

$$
z=x_{1}+i x_{2}, \quad \bar{z}=x_{1}-i x_{2}
$$

where $i=\sqrt{-1}$ is the imaginary unit. Let also

$$
\Delta=\frac{\partial^{2}}{\partial x_{1}^{2}}+\frac{\partial^{2}}{\partial x_{2}^{2}}
$$

be the Laplace operator in $\mathbb{R}^{2} \cong \mathbb{C}$.

Let $\mathcal{D}$ be the unit disk in $\mathbb{C}$. We consider complex-valued functions defined in $\mathcal{D}$ and its closure $\overline{\mathcal{D}}$. Let $S$ be an (relatively) open connected subset of $\partial \mathcal{D}$ and let $a_{0}, b_{0}, b_{1}, b_{2}$ be non-negative numbers with $b_{1}+b_{2}=2$.

Consider the following (generally, non-coercive, which we explain below) Zaremba type mixed boundary value problem for the Laplace operator in $\mathcal{D}$. Given a distribution $f$ in $\mathcal{D}$, find a distribution $u$ in $\mathcal{D}$ which satisfies

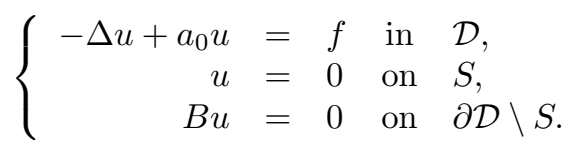

where the boundary operator $B$ is defined as

$$
B u=b_{0} u+b_{1} z \partial+b_{2} \bar{z} \bar{\partial} .
$$

Of course, the case $S=\partial \mathcal{D}$ corresponds to the Dirichlet problem for the Laplace operator in the disk $\mathcal{D}$.

Zaremba [25] studied similar problem in the space of smooth functions over a smooth domain $D$ of the Euclidean space $\mathbb{R}^{3}$ with $S \neq \emptyset, \partial D \backslash S \neq \emptyset$, and $B$ being the (exterior) normal derivative with respect to $\partial D$, i.e.

$$
B u=\frac{\partial u}{\partial \nu}=\nu_{1} \frac{\partial u}{\partial x_{1}}+\nu_{2} \frac{\partial u}{\partial x_{2}}+\nu_{3} \frac{\partial u}{\partial x_{3}}
$$

(here $\nu=\left(\nu_{1}, \nu_{2}, \nu_{3}\right)$ is the unit normal vector with respect to $\partial D$ ) which corresponds to $b_{0}=0, b_{1}=b_{2}=1$ in our case of the unit disk on the complex plane.

To specify the functional space for solutions to (2.1) we use the standard method of Hermitian forms. Namely, one usually defines a Hermitian form $h(\cdot, \cdot)$ on a suitable space Hilbert $H$ and reformulates $(2.1)$ in the following way: given $f$ in the dual $H^{\prime}$ for the space $H$, find $u \in H$ satisfying

$$
h(u, v)=\langle f, v\rangle \text { for all } v \in H
$$


where $\langle\cdot, \cdot\rangle$ is a pairing between $H$ and $H^{\prime}$. Recall that a Hermitian form $h(u, v)$ is called coercive on a Hilbert space $H$ if

$$
h(u, u) \geq\|u\|_{H}^{2} \text { for all } u \in H
$$

with a constant $c>0$ independent on $u$. Typically, if the form $h(\cdot, \cdot)$ is not coercive then a loss of regularity is expected for solutions of the problem induced by the form. In this case the problem (2.2) is called non-coercive. However, in many situations one may successfully use non-coercive forms to study boundary value problems (see, for instance, [11], [19], [20]). We follow these examples.

With this purpose, let $\bar{\partial}$ stand for the Cauchy-Riemann operator, corresponding to the complex structure, i.e.

$$
\bar{\partial}=\frac{1}{2}\left(\frac{\partial}{\partial x_{1}}+i \frac{\partial}{\partial x_{2}}\right) .
$$

The formal adjoint $\bar{\partial}^{*}$ of $\bar{\partial}$ is the operator

$$
-\frac{1}{2}\left(\frac{\partial}{\partial x_{1}}-i \frac{\partial}{\partial x_{2}}\right)=:-\partial .
$$

An easy computation shows that $\bar{\partial}^{*} \bar{\partial}$ just amounts to the $-1 / 4$ multiple of the Laplace operator in the real plane $\mathbb{R}^{2}$ with the coordinates $x=\left(x_{1}, x_{2}\right)$.

As usual, we write $L^{2}(\mathcal{D})$ for the space of all the measurable functions $u$ in $\mathcal{D}$, such that the integral of $|u|^{2}$ over $\mathcal{D}$ is finite. For functions $u \in C^{1}(\overline{\mathcal{D}})$ we introduce the norm

$$
\|u\|_{H^{1}(\mathcal{D})}=\left(\int_{\mathcal{D}} \sum_{j=1}^{n}\left|\partial_{j} u\right|^{2} d x+\int_{\mathcal{D}}|u|^{2} d x\right)^{1 / 2},
$$

where $\partial_{j}=\frac{\partial}{\partial x_{j}}$. The completion of the space $C^{1}(\overline{\mathcal{D}})$ with respect to this norm is the Sobolev space $H^{1}(\mathcal{D})$. It is known that $H^{1}(\mathcal{D})$ is a Hilbert space with inner product

$$
(u, v)_{H^{1}(\mathcal{D})}=\int_{\mathcal{D}} \sum_{j=1}^{n} \partial_{j} u \overline{\partial_{j} v} d x,
$$

for $u, v \in H^{1}(\mathcal{D})$.

Consider the Hermitian form

$$
(u, v)_{+}=a_{0}(u, v)_{L^{2}(\mathcal{D})}+2 b_{1}(\partial u, \partial v)_{L^{2}(\mathcal{D})}+2 b_{2}(\bar{\partial} u, \bar{\partial} v)_{L^{2}(\mathcal{D})}+b_{0}(u, v)_{L^{2}(\partial \mathcal{D} \backslash S)} .
$$

Clearly, the Hermitian form $(u, v)_{+}$is coherent with the functional

$$
\|u\|_{+}=\left(a_{0}\|u\|_{L^{2}(\mathcal{D})}^{2}+2 b_{1}\|\partial u\|_{L^{2}(\mathcal{D})}^{2}+2 b_{2}\|\bar{\partial} u\|_{L^{2}(\mathcal{D})}^{2}+b_{0}\|u\|_{L^{2}(\partial \mathcal{D} \backslash S)}^{2}\right)^{1 / 2}
$$

on $H^{1}(\mathcal{D})$. Let $H^{1}(\mathcal{D}, S)$ be the closed subspace in $H^{1}(\mathcal{D})$ consisting of functions vanishing on $S$. If the functional defines a norm on $H^{1}(\mathcal{D}, S)$, we denote by $H^{+}(\mathcal{D})$ the completion of $H^{1}(\mathcal{D}, S)$ with respect to this norm. Then $H^{+}(\mathcal{D})$ is actually a Hilbert space with inner product $(u, v)_{+}$. 
By the definition, each element of the space $H^{+}(\mathcal{D})$ has a well defined trace on $\partial \mathcal{D}$ belonging to $L^{2}(\partial \mathcal{D})$; in particular, by the elliptic regularity the functions from $H^{+}(\mathcal{D})$ belong to $H_{\text {loc }}^{1}(\mathcal{D} \cup S)$ and equal to zero on $S$.

Let us assume that $H^{+}(\mathcal{D})$ is continuously embedded to $L^{2}(\mathcal{D})$. If we denote by $H^{-}(\mathcal{D})$ the dual to the space $H^{+}(\mathcal{D})$ and by $\langle\cdot, \cdot\rangle$ the pairing induced by the inner product in $L^{2}(\mathcal{D})$ then, using integration by parts and the fact that

$$
\begin{gathered}
4 \partial \bar{\partial}=4 \bar{\partial} \partial=\Delta, \\
(z \partial u, v)_{L^{2}(\partial \mathcal{D} \backslash S)}=2(\partial u, \partial v)_{L^{2}(\mathcal{D})}+1 / 2(\Delta u, v)_{L^{2}(\mathcal{D})}, \\
(\bar{z} \bar{\partial} u, v)_{L^{2}(\partial \mathcal{D} \backslash S)}=2(\bar{\partial} u, \bar{\partial} v)_{L^{2}(\mathcal{D})}+1 / 2(\Delta u, v)_{L^{2}(\mathcal{D})}
\end{gathered}
$$

for all $u, v \in H^{2}(\mathcal{D}) \cap H^{1}(\mathcal{D}, S)$, we see that the Zaremba type mixed boundary value problem for the Laplace operator in $\mathcal{D}$ reads as follows.

Problem 2.1. Given $f \in H^{-}(\mathcal{D})$, find $u \in H^{+}(\mathcal{D})$ which satisfies

$$
(u, v)_{+}=\langle f, v\rangle \text { for all } v \in H^{+}(\mathcal{D}) .
$$

Then the Riesz Theorem on the general form for continuous linear functionals on Hilbert spaces provides a unique solution $u \in H^{+}(\mathcal{D})$ to the Problem 2.1 for each $f \in H^{-}(\mathcal{D})$ "orthogonal" to the null space of the problem with respect to the pairing $\langle\cdot, \cdot\rangle$, see, for instance, [13, Ch. 4] and elsewhere. According to the Uniqueness Theorem for the Cauchy problem for solutions to elliptic systems ([18, Theorem 2.8]), the null-space of the problem equals to zero if $S \neq \emptyset$. Actually, to look on the eigenfunctions and the eigenvalues of Problem 2.1, it is convenient to consider the bounded linear continuously invertible operator $L: H^{+}(D) \rightarrow H^{-}(D)$, induced by $(2.3)$.

Example 2.2. If $b_{1}>0$ and $b_{2}>0$, then one easily conclude that the norm $\|\cdot\|_{+}$is equivalent to the standard norm $\|\cdot\|_{H^{1}(\mathcal{D})}$ on $H^{1}(\mathcal{D}, S)$ and the space $H^{+}(\mathcal{D})$ coincides with $H^{1}(\mathcal{D}, S)$, see, for instance, [13, Ch. 4]. This means that the form $(\cdot, \cdot)_{+}$is coercive and Problem 2.1 satisfies the Shapiro-Lopatinsky conditions on $\partial \mathcal{D} \backslash S$ and hence its solutions belong to $C^{\infty}(\overline{\mathcal{D}} \backslash \partial S) \cap H^{1}(\mathcal{D})$ for any $f \in C^{\infty}(\overline{\mathcal{D}})$. In particular, the Problem 2.1 is Fredholm and its index equals to zero. Moreover, its spectrum is discrete, the eigenvalues are non-negative (and even $\lambda \geq a_{0}$ ) and the corresponding eigenvectors form orthogonal bases in the spaces $H^{+}(\mathcal{D}), H^{-}(\mathcal{D})$ and $L^{2}(\mathcal{D})$ (see, for instance, [13, Ch. 4] and elsewhere). Actually, these are the eigenvectors of the linear compact self-adjoint operators $\iota^{*} \iota L^{-1}: H^{-}(\mathcal{D}) \rightarrow H^{-}(\mathcal{D}), L^{-1} \iota^{*} \iota: H^{+}(\mathcal{D}) \rightarrow$ $H^{+}(\mathcal{D})$, and $\iota L^{-1} \iota^{*}: L^{2}(\mathcal{D}) \rightarrow L^{2}(\mathcal{D})$ where $\iota: H^{+}(D) \rightarrow L^{2}(D)$ is the natural embedding and $\iota^{*}: L^{2}(D) \rightarrow H^{-}(D)$ is the adjoint operator for $\iota$, see, for instance, [19, Lemma 3.1].

Example 2.3. If $b_{0}>0$ and one of the numbers $b_{j}>0, j=1,2$, is positive, then the norm $\|\cdot\|_{+}$is not weaker than the standard norm $\|\cdot\|_{H^{1 / 2}(\mathcal{D})}$ on $H^{1}(\mathcal{D}, S)$ and the space $H^{+}(\mathcal{D})$ is continuously embedded to the SobolevSlobodetskii space $H^{1 / 2}(\mathcal{D}, S)$, see [19, Theorem 2.5]. The example constructed in [19, Remark 5.1] shows that the embedding is sharp if $b_{1}=0$ 
and thus the form $(\cdot, \cdot)_{+}$is not coercive in this case. On the other hand, if $b_{1}=0$ then Problem 2.1 is a boundary value problem with the so-called $\bar{\partial}$ Neumann boundary condition. Thus combining [19, Theorem 2.5] and results of [11] we conclude that its solutions belong to $C^{\infty}(\overline{\mathcal{D}} \backslash \partial S) \cap H^{1 / 2}(\mathcal{D})$ for any $f \in C^{\infty}(\overline{\mathcal{D}})$. Again, the Problem 2.1 is Fredholm, its index equals to zero, its spectrum is discrete, the eigenvalues are non-negative (and even $\lambda \geq a_{0}$ ) and the corresponding eigenvectors form orthogonal bases in the spaces $H^{+}(\mathcal{D})$, $H^{-}(\mathcal{D})$ and $L^{2}(\mathcal{D})$ (see, for instance, [19, Lemma 3.1]).

Example 2.4. If $S=\emptyset, a_{0}>0$ and $b_{0}=b_{1}=0$ then the null space of Problem 2.1 equals to the space of the holomorphic functions of the class $L^{2}(\mathcal{D})$ and hence in this case the Problem 2.1 is not Fredholm.

In the next sections we will be concentrated on finding the eigenvalues and the eigenfunctions for Problem 2.1. Since the spectrum of the Dirichlet problem is well known, we will consider the case where $S \neq \partial \mathcal{D}$ only. We will pay almost no attention to the non-Fredholm case $b_{0}=b_{1} \cdot b_{2}=0$.

\section{Applying the Fourier method}

Consider the mixed problem

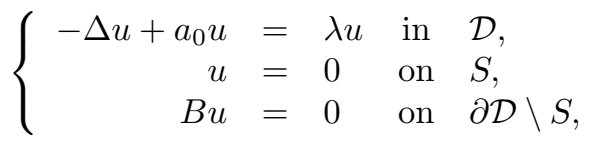

in the space $H^{+}(\mathcal{D})$, i.e.

$$
2 b_{1}(\partial u, \partial v)_{L^{2}(\mathcal{D})}+2 b_{2}(\bar{\partial} u, \bar{\partial} v)_{L^{2}(\mathcal{D})}+b_{0}(u, v)_{L^{2}(\partial \mathcal{D} \backslash S)}=\left(\lambda-a_{0}\right)(u, v)_{L^{2}(\mathcal{D})}
$$

for all $v \in H^{1}(\mathcal{D}, S)$.

As we have already noted, we have to study the case $a_{0} \leq \lambda \in \mathbb{R}$ only. Since the Helmholtz operator $\left(-\Delta+a_{0}-\lambda\right)$ is elliptic, we see that the solutions to (3.1) belongs to $C^{\infty}(\mathcal{D} \cup S)$. Moreover, according to Petrovskii Theorem they are real analytic in $\mathcal{D}$. The results by C. B. Morrey and L. Nirenberg [14] imply that the solutions also extends analytically in a neighbourhood of any compact $K$ from the (relative) interior of $S$ on $\partial \mathcal{D}$. However the points of $\partial S \subset \partial \mathcal{D}$ can be singular for the solutions to (3.1).

Let

$$
\mathcal{J}_{p}(z)=\sum_{k=0}^{\infty} \frac{(-1)^{k}}{2^{2 k+p}} \frac{z^{2 k+p}}{k !(k+p) !}, \quad \mathcal{J}_{-p}(z)=(-1)^{p} \mathcal{J}_{p}(z), z \in \mathbb{C}, p \in \mathbb{Z}_{+},
$$

be the Bessel functions, see, for example, [24, §2.11].

For the coercive case the following proposition is well known, see [23, Suppl. II, P. 1, §2]. 
Proposition 3.1. Let $S=\emptyset$ and $b_{0}^{2}+\left(b_{1} \cdot b_{2}\right)^{2} \neq 0$. Then the complete system of solutions to (3.1) in the space $\mathrm{H}^{+}(\mathcal{D})$ consists of functions of the form $u_{p}(r, \varphi)=g_{p}(r) \exp [i p \varphi]=\exp [i p \varphi] u_{p}(z)=(z /|z|)^{p} \mathcal{J}_{p}\left(|z| \sqrt{\lambda_{p}-a_{0}}\right), p \in \mathbb{Z}$, where the corresponding eigenvalue $\lambda_{p}$ is a root of the function

$$
G_{p}(\lambda)=\left(b_{0}-p b_{2}\right) \mathcal{J}_{p}\left(\sqrt{\lambda-a_{0}}\right)+\sqrt{\lambda-a_{0}} \mathcal{J}_{p-1}\left(\sqrt{\lambda-a_{0}}\right) .
$$

Proof. We may apply the Fourier method of separation of variables. Actually, the matter is quite similar to the coercive mixed problem for the Laplace operator in the ball (see [23, Suppl. II, P. 1, §2]).

To this end, we pass to the polar coordinates

$$
\left\{\begin{array}{l}
x_{1}=r \cos \varphi \\
x_{2}=r \sin \varphi
\end{array}\right.
$$

where $\varphi \in[0,2 \pi]$ are coordinates on the unit circle $\partial \mathcal{D}$ in $\mathbb{C}$. The Laplace operator $\Delta$ in the spherical coordinates takes the form

$$
\Delta=\frac{1}{r^{2}}\left(\left(r \frac{\partial}{\partial r}\right)^{2}+\frac{\partial^{2}}{\partial \varphi^{2}}\right) .
$$

On the other hand, in the unit disk we have

$$
\frac{\partial}{\partial \nu}=r \frac{\partial}{\partial r}, \quad \bar{z} \bar{\partial}=\frac{1}{2}\left(r \frac{\partial}{\partial r}+i \frac{\partial}{\partial \varphi}\right), \quad z \partial=\frac{1}{2}\left(r \frac{\partial}{\partial r}-i \frac{\partial}{\partial \varphi}\right) .
$$

It follows from (3.6) that

$$
B u=b_{0} u+\frac{b_{2}-b_{1}}{2} i \frac{\partial u}{\partial \varphi}+r \frac{\partial u}{\partial r} .
$$

As usual, to solve the homogeneous equation $\left(-\Delta+a_{0}-\lambda\right) u=0$, we write $u(r, \varphi)=g(r) h(\varphi)$, obtaining two separate equations for $g$ and $h$. Namely,

$$
\begin{aligned}
\left(-\left(r \frac{\partial}{\partial r}\right)^{2}+\left(a_{0}-\lambda\right) r^{2}\right) g & =c g, \\
-\frac{\partial^{2} h}{\partial \varphi^{2}} & =c h
\end{aligned}
$$

$c$ being an arbitrary constant.

The second equation has non-zero solutions if and only if $c$ is an eigenvalue of $\left(-\frac{\partial^{2}}{\partial \varphi^{2}}\right)$. These are well known to be $c=p^{2}$, for $p=\mathbb{Z}$ (see, for instance, [23, Suppl. II, P. 1, §2]). The corresponding eigenfunctions of $\left(-\frac{\partial^{2}}{\partial \varphi^{2}}\right)$ are the complex exponents

$$
h_{p}(\varphi)=\exp [i p \varphi] .
$$

Clearly,

$$
h_{p}=\left\{\begin{array}{lll}
z_{\mid \partial \mathcal{D}}^{p} & \text { if } & p \in \mathbb{Z}_{+} \\
\bar{z}_{\mid \partial \mathcal{D}}^{p} & \text { if } & -p \in \mathbb{Z}_{+}
\end{array}\right.
$$


It follows from (3.6) that

$$
B u=h_{p}(\varphi)\left(\frac{\left(2 b_{0}+\left(b_{1}-b_{2}\right) p\right) g(r)}{2}+r g^{\prime}(r)\right) .
$$

Fix $p \in \mathbb{Z}$ and consider the following Sturm-Liouville Problem for ordinary differential equation with respect to the variable $r, 0<r<1$ (see, for instance, [23, Suppl. II, P. 1, §2]),

$$
\left(-\frac{1}{r^{2}}\left(r \frac{\partial}{\partial r}\right)^{2}+\frac{p^{2}}{r^{2}}\right) g_{p}(r)=\left(\lambda-a_{0}\right) g_{p}(r)
$$

$g_{p}^{\prime}(1)+\left(\frac{2 b_{0}+\left(b_{1}-b_{2}\right) p}{2}\right) g_{p}(1)=0$ and $g_{p}(r)$ is bounded at the point $r=0$.

Actually, if $\lambda \in \mathbb{R}$ then (3.7) is a version of the Bessel equation, and its (real-valued) solution $g(r)$ can be expressed via the Bessel function $\mathcal{J}_{p}$ while the space of all the solutions is two-dimensional, see, for instance, [23, Suppl. II, P. 1, §2]. For example, if $\lambda=a_{0}$ then $g_{p}(r)=a r^{p}+b r^{-p}$ with arbitrary constants $a$ and $b$ is the general solution to (3.7). In the general case the space of solutions to (3.7) contains a one-dimensional subspace of functions bounded at the point $r=0$, cf. [23, Suppl. II, P. 1, §2]. More precisely,

$$
g_{p}(r)=\mathcal{J}_{p}\left(r \sqrt{\lambda-a_{0}}\right) \text {. }
$$

For $p \in \mathbb{Z}$, fix a non-trivial solution $g_{p}(r)$ to (3.7), (3.8) corresponding to an eigenvalue $\lambda_{p}$. It is known that the system $\left\{g_{p}\right\}_{p \in \mathbb{Z}}$ form an orthogonal basis in the space $L^{2}([0,1], r)$ with the weight $r$. Then the function $u_{p}=$ $g_{p}(r) \exp [i p \varphi]$ satisfies

$$
\begin{gathered}
\left(-\Delta+\left(a_{0}-\lambda_{p}\right)\right) u_{p}=0 \text { on } \mathbb{C}, \\
B u_{p}=0 \text { on } \partial \mathcal{D} .
\end{gathered}
$$

Indeed, by (3.5), (3.6), (3.7) and the discussion above we conclude that this equality holds in $\mathbb{C} \backslash\{0\}$. We now use the fact that $u_{p}$ is bounded at the origin to see that (3.9) holds. On the other hand, (3.10) follows from (3.6) immediately. Then $u_{p}$ is given by (3.3). The corresponding eigenvalue $\lambda_{p}$ is the root of the function

$$
G_{p}(\lambda)=\sqrt{\lambda-a_{0}} \mathcal{J}_{p}^{\prime}\left(\sqrt{\lambda-a_{0}}\right)+\left(\frac{2 b_{0}+\left(b_{1}-b_{2}\right) p}{2}\right) \mathcal{J}_{p}\left(\sqrt{\lambda-a_{0}}\right) .
$$

By the famous differential relations (see, for instance, $[24, \S 2.12]$ or $[1,9.1 .27]$ )

$$
\mathcal{J}_{p}^{\prime}(r)=\mathcal{J}_{p-1}(r)-\frac{p}{r} \mathcal{J}_{p}(r)
$$

and hence $G_{p}$ is given by (3.4).

As usual, the completeness of the system $\left\{u_{p}\right\}_{p \in \mathbb{Z}}$ in the space $L^{2}(\mathcal{D})$ follows from the Fubini theorem. Indeed, since the system $\{\exp [i p \varphi]\}_{p \in \mathbb{Z}}$ is an orthogonal basis in the space $L^{2}([0,2 \pi])$ and the system $\left\{g_{p}(r)\right\}_{p \in \mathbb{Z}}$ form an orthogonal basis in the space $L^{2}([0,1], r)$, the familiar arguments show that the system $\left\{g_{p}(r) \exp [i p \varphi]\right\}_{p \in \mathbb{Z}}$ is an orthogonal basis in the space $L^{2}([0,2 \pi] \times[0,1])=L^{2}(\mathcal{D})$. 
Remark 3.2. We emphasize that the scheme works even in the cases where we can not guarantee a compact embedding in $H^{+}(\mathcal{D}) \hookrightarrow L^{2}(\mathcal{D})$. Indeed, if $S=\emptyset, a_{0}>0, b_{0}=b_{1}=0$ then the embedding $H^{+}(\mathcal{D}) \hookrightarrow L^{2}(\mathcal{D})$ is continuous. It can not be compact because the space $H^{+}(\mathcal{D})$ contains the space of holomorhic $L^{2}(\mathcal{D})$-functions. However, using the problem (3.7), (3.8) for the Bessel equation, we can construct a basis in $L^{2}(\mathcal{D})$, consisting of eigenfunctions of the corresponding Zaremba problem. Note that if $\lambda=a_{0}$ then all the holomorphic monomials $\left\{z^{p}\right\}_{p \in \mathbb{Z}_{+}}$are eigenvectors corresponding to this eigenvalue with infinite multiplicity.

Proposition 3.3. Let the (relative) interiors of the sets $S$ and $\partial \mathcal{D} \backslash S$ on $\partial \mathcal{D}$ be not empty. If either $a_{0}>0$ or $b_{0}>0$ then solutions to (3.2), belonging to the space $H^{+}(\mathcal{D})$, can not be presented as finite sums of the type

$$
u(r, \varphi)=\sum_{k=1}^{N} c_{k} e^{i p_{k} \varphi} \mathcal{J}_{p_{k}}\left(r \sqrt{\lambda-a_{0}}\right)
$$

with some numbers $N \in \mathbb{N}, p_{k} \in \mathbb{Z}$ and $c_{k} \in \mathbb{C}$.

Proof. Equation (3.2) implies that the solution $u$, corresponding to $\lambda=a_{0}$, is a solution to elliptic system

$$
b_{1} \partial u=b_{2} \bar{\partial} u=0 \text { in } \mathcal{D}
$$

If either $a_{0}>0$ or $b_{0}>0$ then $u \in L^{2}(\mathcal{D})$ and hence it has a finite order of growth near $\partial \mathcal{D}$. Since $u$ vanishes on $S$ in the weak sense, the Uniqueness Theorem for the Cauchy problem for elliptic equations, see, for instance, [18, Theorem 2.8], implies that $u \equiv 0$ in $\mathcal{D}$.

Fix $\lambda>a_{0}$. Assume that $u$ is presented in the form (3.11). Then

$$
-\Delta u=\lambda u \text { in } \mathcal{D} \text {. }
$$

Applying the boundary operator on $\partial \mathcal{D} \backslash S$ we obtain

$$
\sum_{k=1}^{N} c_{k} e^{i p_{k} \varphi}\left(\frac{\left(2 b_{0}+\left(b_{1}-b_{2}\right) p_{k}\right)}{2} \mathcal{J}_{p_{k}}\left(\sqrt{\lambda-a_{0}}\right)+\sqrt{\lambda-a_{0}} \mathcal{J}_{p_{k}}^{\prime}\left(\sqrt{\lambda-a_{0}}\right)\right)=0 .
$$

Since any finite system $\left\{e^{i p_{k} \varphi}\right\}_{k=1}^{N}$ is linearly independent on any interval $(\alpha, \beta) \subset[0,2 \pi]$, we see that

$$
\left(\frac{\left(2 b_{0}+\left(b_{1}-b_{2}\right) p_{k}\right)}{2} \mathcal{J}_{p_{k}}\left(\sqrt{\lambda-a_{0}}\right)+\sqrt{\lambda-a_{0}} \mathcal{J}_{p_{k}}^{\prime}\left(\sqrt{\lambda-a_{0}}\right)\right)=0
$$

for all $1 \leq k \leq N$ and hence $B u=0$ on the whole boundary $\partial \mathcal{D}$.

Since $u$ should also vanish on $S$, we see that again the Uniqueness Theorem for the Cauchy problem for elliptic equations, see, for instance, $[18$, Theorem 2.8], implies that $u \equiv 0$ in $\mathcal{D}$.

This proposition means that, for $S \neq \emptyset, S \neq \partial \mathcal{D}$, we should look for the eigenfunctions in the form

$$
u(r, \varphi)=\sum_{k=1}^{\infty} c_{k} e^{i p_{k} \varphi} \mathcal{J}_{p_{k}}\left(r \sqrt{\lambda-a_{0}}\right)
$$


with some numbers $p_{k} \in \mathbb{Z}$ and $c_{k} \in \mathbb{C}$ or even

$$
u(r, \varphi)=\int_{-\infty}^{+\infty} e^{i p \varphi} \mathcal{J}_{p}\left(r \sqrt{\lambda-a_{0}}\right) d \mu(p)
$$

with some measure $d \mu$ on $\mathbb{R}$.

The last formula suggests us to use the theorem on the exponential representation of solutions to partial differential equations with constant coefficients (see [15, Introduction, p. 19]) instead of the method of separation of variables.

\section{Applying the theorem on the exponential representation}

From now on we set $a_{0}=0$, because a non-zero $a_{0}$ produces a shift of the spectrum only.

Applying the Fourier transform we see that the characteristic manifold of the Helmholtz operator $(-\Delta-\lambda), \lambda \geq 0$, coincides with the circle

$$
\mathcal{N}=\left\{y \in \mathbb{R}^{2}: y_{1}^{2}+y_{2}^{2}=\lambda\right\}=\left\{\zeta \in \mathbb{C}:|\zeta|^{2}=\lambda\right\},
$$

where $\zeta=y_{1}+i y_{2}$.

By the definition and [19, Theorem 2.5], if $b_{0}^{2}+\left(b_{1} \cdot b_{2}\right)^{2} \neq 0$ then each element of the space $H^{+}(\mathcal{D})$ has a well defined trace on $\partial \mathcal{D}$ belonging to $L^{2}(\partial \mathcal{D})$. In particular, the solutions to $(3.1)$ in the spaces $H^{+}(\mathcal{D})$ are smooth functions in the disk $\mathcal{D}$ having a finite order of growth near $\partial \mathcal{D}$, see [18].

Then the theorem on the exponential representation of solutions to partial differential equations with constant coefficients [15, Introduction and Ch. VI, $\S 4$, theorem 1] suggests us to look for the solution to (3.1) in the following form

$$
u(x)=\int_{y_{1}^{2}+y_{2}^{2}=\lambda} \exp \left[i\left(x_{1} y_{1}+x_{2} y_{2}\right)\right] d \mu(y), \lambda>0 .
$$

where $d \mu(y)$ is a (not uniquely defined, in general) complex measure on $\mathcal{N}$, i.e. $d \mu \in C^{\prime}(\overline{\mathcal{N}})$, where $C^{\prime}(\overline{\mathcal{N}})$ is the dual to the Banach space $C(\overline{\mathcal{N}})$ of continuous functions on $\overline{\mathcal{N}}$. After a complexification we get a slightly different form:

$$
u(z)=\int_{|\zeta|=1} \exp [\sqrt{-\lambda}(z \bar{\zeta}+\bar{z} \zeta) / 2] d \mu(\zeta), \lambda>0 .
$$

Since the solutions (3.1) in the spaces $H^{+}(\mathcal{D})$ are smooth functions on the disk $\mathcal{D}$ having a finite order of growth near $\partial \mathcal{D}$, we conclude that the traces (or, more precisely, weak boundary values) of $B u$ are also well defined in the space of distributions on $\partial \mathcal{D}$, see $[18$, Theorem 2.6]. In particular, since $u_{\mid \partial \mathcal{D} \backslash S} \in L^{2}(\partial \mathcal{D} \backslash S)$ we conclude that $b_{1} z \partial u+b_{2} \bar{z} \bar{\partial} u$ belongs to $L^{2}(\partial \mathcal{D} \backslash S)$ for any solution to $(3.1)$ belonging to $H^{+}(\mathcal{D})$.

Since $u \in L^{2}(S)$ and $B u \in L^{2}(\partial \mathcal{D} \backslash S)$ we may look for a measure $d \mu(\zeta)$ such that $u$ presented by (4.1) has traces of $u$ and (weak boundary values of) $B u$ vanishing on $S$ and $(\partial \mathcal{D} \backslash S)$, respectively, as elements of the Lebesgue spaces $L^{2}(S)$ and $L^{2}(\partial \mathcal{D} \backslash S)$. 
With this purpose, we assume that

$$
S=\{z \in \mathbb{C}:|z|=1, \alpha<\arg (z)<2 \pi\},
$$

with a number $\alpha, 0<\alpha \leq 2 \pi(\alpha=2 \pi$ corresponds to the case $S=\emptyset)$. Then the system $\left\{z^{2 p \pi /(2 \pi-\alpha)}\right\}_{p \in \mathbb{Z}}$ is an orthogonal basis in $L^{2}(S)$ if $0<\alpha<2 \pi$. Hence

$$
\partial \mathcal{D} \backslash S=\{z \in \mathbb{C}:|z|=1,0 \leq \arg (z) \leq \alpha\}
$$

and the system $\left\{z^{2 p \pi / \alpha}\right\}_{p \in \mathbb{Z}}$ is an orthogonal basis in $L^{2}(\partial \mathcal{D} \backslash S)$.

Now a function $u$ of the form (4.1) is a solution to (3.1), belonging to $H^{+}(\mathcal{D})$, if and only if for all $p \in \mathbb{Z}$ we have

$$
\begin{gathered}
\int_{S} u(z) z^{-2 p \pi /(2 \pi-\alpha)} \frac{d z}{z}=0 \\
\int_{\partial \mathcal{D} \backslash S}(B u)(z) z^{-2 p \pi / \alpha} \frac{d z}{z}=0,
\end{gathered}
$$

where

$$
B u(z)=\int_{|\zeta|=1}\left(b_{0}+\frac{\sqrt{-\lambda} b_{1} z \bar{\zeta}}{2}+\frac{\sqrt{-\lambda} b_{2} \bar{z} \zeta}{2}\right) \exp [\sqrt{-\lambda}(z \bar{\zeta}+\bar{z} \zeta) / 2] d \mu(\zeta)
$$

Since

$$
\exp [z+\bar{z}]=\sum_{m=0}^{\infty} \sum_{j=0}^{m} \frac{z^{j} \bar{z}^{m-j}}{j !(m-j) !},
$$

equalities (4.2) and (4.3) are true for all $p \in \mathbb{Z}$ if and only if for all $p \in \mathbb{Z}$ we have

$$
\begin{gathered}
\int_{S} \int_{|\zeta|=1} \sum_{m=0}^{\infty}\left(\frac{\sqrt{-\lambda}}{2}\right)^{m} \sum_{j=0}^{m} \frac{z^{j-2 p \pi /(2 \pi-\alpha)} \bar{z}^{m-j} \bar{\zeta}^{j} \zeta^{m-j}}{j !(m-j) !} d \mu(\zeta) \frac{d z}{z}=0, \quad \text { (4.6) } \\
b_{0} \int_{\partial \mathcal{D} \backslash S} \int_{|\zeta|=1} \sum_{m=0}^{\infty}\left(\frac{\sqrt{-\lambda}}{2}\right)^{m} \sum_{j=0}^{m} \frac{z^{j-2 p \pi / \alpha} \bar{z}^{m-j} \bar{\zeta}^{j} \zeta^{m-j}}{j !(m-j) !} d \mu(\zeta) \frac{d z}{z}+ \\
b_{1} \int_{\partial \mathcal{D} \backslash S} \int_{|\zeta|=1} \sum_{m=0}^{\infty}\left(\frac{\sqrt{-\lambda}}{2}\right)^{m+1} \sum_{j=0}^{m} \frac{z^{j+1-2 p \pi / \alpha} \bar{z}^{m-j} \bar{\zeta}^{j+1} \zeta^{m-j}}{j !(m-j) !} d \mu(\zeta) \frac{d z}{z}+ \\
b_{2} \int_{\partial \mathcal{D} \backslash S} \int_{|\zeta|=1} \sum_{m=0}^{\infty}\left(\frac{\sqrt{-\lambda}}{2}\right)^{m+1} \sum_{j=0}^{m} \frac{z^{j-2 p \pi / \alpha} \bar{z}^{m-j+1} \bar{\zeta}^{j} \zeta^{m-j+1}}{j !(m-j) !} d \mu(\zeta) \frac{d z}{z}=0 .
\end{gathered}
$$

Recall that

$$
\int_{\beta_{1} \leq \arg (z) \leq \beta_{2}} z^{p} \bar{z}^{q} \frac{d z}{z}= \begin{cases}i\left(\beta_{2}-\beta_{1}\right), & p=q \\ \frac{\exp \left[i \beta_{2}(p-q)\right]-\exp \left[i \beta_{1}(p-q)\right]}{p-q}, & p \neq q .\end{cases}
$$


Hence, using (4.8) we see that (4.2) is true for all $p \in \mathbb{Z}$ if and only if for all $p \in \mathbb{Z}$ we have

$$
\begin{aligned}
0= & \int_{|\zeta|=1} \sum_{\substack{\frac{m}{2}+\frac{p \pi}{2 \pi-\alpha} \in \mathbb{Z}_{+} \\
0 \leq \frac{m}{2}+\frac{p \pi}{2 \pi-\alpha} \leq m}}\left(\frac{\sqrt{-\lambda}}{2}\right)^{m} \frac{(2 \pi-\alpha) i \zeta^{\frac{-2 p \pi}{2 \pi-\alpha}} d \mu(\zeta)}{\left(\frac{m}{2}-\frac{p \pi}{2 \pi-\alpha}\right) !\left(\frac{m}{2}+\frac{p \pi}{2 \pi-\alpha}\right) !}+ \\
& \int_{|\zeta|=1} \sum_{m=0}^{\infty}\left(\frac{\sqrt{-\lambda}}{2}\right)^{m} \sum_{\substack{0 \leq j \leq m \\
0 \leq-\frac{2 p \pi}{2 \pi-\alpha} \neq m}} \mathcal{K}_{m, j}^{(1)}(p, \alpha) \frac{\bar{\zeta}^{j} \zeta^{m-j} d \mu(\zeta)}{j !(m-j) !}
\end{aligned}
$$

where

$$
\mathcal{K}_{m, j}^{(1)}(p, \alpha)=\frac{\exp \left[i 2 \pi\left(2 j-\frac{2 p \pi}{2 \pi-\alpha}-m\right)\right]-\exp \left[i \alpha\left(2 j-\frac{2 p \pi}{2 \pi-\alpha}-m\right)\right]}{2 j-\frac{2 p \pi}{2 \pi-\alpha}-m} .
$$

Similarly, using (4.7) and (4.8) we see that (4.3) is true for all $p \in \mathbb{Z}$ if and only if for all $p \in \mathbb{Z}$ we have

$$
\begin{aligned}
& 0=b_{0} \int_{|\zeta|=1} \sum_{\substack{\frac{m}{2}+\frac{p \pi}{\alpha} \in \mathbb{Z}_{+} \\
0 \leq \frac{m}{2}+\frac{p \pi}{\alpha} \leq m}}\left(\frac{\sqrt{-\lambda}}{2}\right)^{m} \frac{\alpha i \zeta^{\frac{-2 p \pi}{\alpha}} d \mu(\zeta)}{\left(\frac{m}{2}-\frac{p \pi}{\alpha}\right) !\left(\frac{m}{2}+\frac{p \pi}{\alpha}\right) !}+ \\
& b_{0} \int_{|\zeta|=1} \sum_{m=0}^{\infty}\left(\frac{\sqrt{-\lambda}}{2}\right)^{m} \sum_{\substack{0 \leq j \leq m \\
2 j-\frac{2 p \pi}{\alpha} \neq m}} \mathcal{K}_{m, j}^{(2)}(p, \alpha) \frac{\bar{\zeta}^{j} \zeta^{m-j} d \mu(\zeta)}{j !(m-j) !}+ \\
& b_{1} \int_{|\zeta|=1} \sum_{\substack{\frac{m-1}{2}+\frac{p \pi}{\alpha} \in \mathbb{Z}_{+} \\
0 \leq \frac{m-1}{2}+\frac{p \pi}{\alpha} \leq m}}\left(\frac{\sqrt{-\lambda}}{2}\right)^{m+1} \frac{\alpha i \zeta^{\frac{-2 p \pi}{\alpha}} d \mu(\zeta)}{\left(\frac{m}{2}-\frac{1}{2}+\frac{p \pi}{\alpha}\right) !\left(\frac{m}{2}+\frac{1}{2}-\frac{p \pi}{\alpha}\right) !}+ \\
& b_{1} \int_{|\zeta|=1} \sum_{m=0}^{\infty}\left(\frac{\sqrt{-\lambda}}{2}\right)^{m+1} \sum_{\substack{0 \leq j \leq m \\
2 j-\frac{2 p \pi}{\alpha} \neq m-1}} \mathcal{K}_{m-1, j}^{(2)}(p, \alpha) \frac{\bar{\zeta}^{j+1} \zeta^{m-j} d \mu(\zeta)}{j !(m-j) !}+ \\
& b_{2} \int_{|\zeta|=1} \sum_{\substack{\frac{m+1}{2}+\frac{p \pi}{\alpha} \in \mathbb{Z}_{+} \\
0 \leq \frac{m+1}{2}+\frac{p \pi}{\alpha} \leq m}}\left(\frac{\sqrt{-\lambda}}{2}\right)^{m+1} \frac{\alpha i \zeta^{-\frac{2 p \pi}{\alpha}} d \mu(\zeta)}{\left(\frac{m+1}{2}+\frac{p \pi}{\alpha}\right) !\left(\frac{m-1}{2}-\frac{p \pi}{\alpha}\right) !}+ \\
& b_{2} \int_{|\zeta|=1} \sum_{m=0}^{\infty}\left(\frac{\sqrt{-\lambda}}{2}\right)^{m+1} \sum_{\substack{0 \leq j \leq m \\
2 j-\frac{2 p \pi}{\alpha} \neq m+1}} \mathcal{K}_{m+1, j}^{(2)}(p, \alpha) \frac{\bar{\zeta}^{j} \zeta^{m-j+1} d \mu(\zeta)}{j !(m-j) !}
\end{aligned}
$$

where

$$
\mathcal{K}_{m, j}^{(2)}(p, \alpha)=\frac{\exp \left[i \alpha\left(2 j-\frac{2 p \pi}{\alpha}-m\right)\right]-1}{2 j-\frac{2 p \pi}{\alpha}-m} .
$$

The relations (4.9) and (4.10) seem to be rather bulky. However we may simplify them describing properly the space of measures on $\partial \mathcal{D}$. Indeed, it is well-known that the space of measures on $\partial \mathcal{D}$ is topologically isomorphic to 
the space $C^{\prime}(\partial \mathcal{D})$ dual to the space $C(\partial \mathcal{D})$ of continuous functions on $\partial \mathcal{D}$. Let us give another description of the dual space.

With this purpose we note that the Banach space $C(\partial \mathcal{D})$ can be identified with the space $\tilde{C}[0,2 \pi]$ of continuous functions on the segment $[0,2 \pi]$ with equal values an the ends of the segment:

$$
\tilde{C}[0,2 \pi]=\{u \in C[0,2 \pi]: u(0)=u(2 \pi)\} .
$$

In particular, the Fourier series

$$
\sum_{q \in \mathbb{Z}}\left(u, \zeta^{q}\right)_{L^{2}(\partial \mathcal{D})} \zeta^{q}
$$

converges uniformly on $\partial \mathcal{D}$ (to $u$ ) for each $u \in C(\partial \mathcal{D})$ and

$$
\|u\|_{C(\partial \mathcal{D})}=\max _{|\zeta|=1}\left|\sum_{q \in \mathbb{Z}}\left(u, \zeta^{q}\right)_{L^{2}(\partial \mathcal{D})} \zeta^{q}\right| .
$$

Consider the (linear) space of the formal series

$$
\mathfrak{C}(\partial \mathcal{D})=\left\{d=\sum_{q \in \mathbb{Z}} \frac{d_{q} \zeta^{q} d \zeta}{2 \pi i \zeta},|\zeta|=1\right\}
$$

where $\left\{d_{q}\right\}$ is chosen in such a way that the functional

$$
\|d\|_{-}=\sup _{\substack{v \in C(\partial \mathcal{D}) \\ v \neq 0}} \frac{\left|\sum_{q \in \mathbb{Z}}\left(v, \zeta^{q}\right)_{L^{2}(\partial \mathcal{D})} d_{q}\right|}{\|v\|_{C(\partial \mathcal{D})}}
$$

is finite. If $\|d\|_{-}=0$ then (having in mind $v=z^{p}$ ) we see that

$$
0 \leq\left|\sum_{q \in \mathbb{Z}}\left(\zeta^{p}, \zeta^{q}\right)_{L^{2}(\partial \mathcal{D})} d_{q}\left\|\zeta^{p}\right\|_{C(\partial \mathcal{D})}^{-1}\right|=2 \pi\left|d_{p}\right| \leq\|d\|_{-}=0
$$

for all $p \in \mathbb{Z}$ and thus $d_{p}=0$ for all $p \in \mathbb{Z}$, too. Hence the functional is a norm on $\mathfrak{C}(\partial \mathcal{D})$. It is easy to see that $\mathfrak{C}(\partial \mathcal{D})$ is a Banach space with the norm $\|\cdot\|_{-}$.

Now we may define the pairing between the spaces $\mathfrak{C}(\partial \mathcal{D})$ and $C(\partial \mathcal{D})$. Namely, we set

$$
\langle v, d\rangle=\sum_{q \in \mathbb{Z}}\left(v, \zeta^{q}\right)_{L^{2}(\partial \mathcal{D})} d_{q}
$$

for each $v \in C(\partial \mathcal{D})$ and $d \in \mathfrak{C}(\partial \mathcal{D})$. According the definition of the space $\mathfrak{C}(\partial \mathcal{D})$ we have

$$
|\langle v, d\rangle| \leq\|v\|_{C(\partial \mathcal{D})}\|d\|_{-}
$$

for all $v \in C(\partial \mathcal{D})$ and $d \in \mathfrak{C}(\partial \mathcal{D})$.

Lemma 4.1. The dual space $C^{\prime}(\partial \mathcal{D})$ is topologically isomorphic to the space $\mathfrak{C}(\partial \mathcal{D})$.

That is, for every fixed $d \in \mathfrak{C}(\partial \mathcal{D})$, pairing (4.12) defines a continuous linear functional $f_{d}$ on $C(\partial \mathcal{D})$ and, for each $f \in C^{\prime}(\partial \mathcal{D})$, there is a unique $d \in \mathfrak{C}(\partial \mathcal{D})$ with $f(v)=f_{d}(v)$ for all $v \in C(\partial \mathcal{D})$. Moreover, the linear map $d \mapsto f_{d}$ is an isometry. 
Proof. Cf. [17, Lemma 3.3] for the Sobolev spaces. It follows from (4.13) that for every fixed $d \in \mathfrak{C}(\partial \mathcal{D})$, the formula

$$
f_{d}(v):=\langle v, d\rangle, v \in C(\partial \mathcal{D}),
$$

defines a continuous linear functional $f_{d}$ on $C(\partial \mathcal{D})$, such that

$$
\left\|f_{d}\right\|_{C^{\prime}(\partial \mathcal{D})} \leq\|d\|_{-} .
$$

Moreover, by the very definition of the norm $\|\cdot\|_{-}$,

$$
\left\|f_{d}\right\|_{C^{\prime}(\partial \mathcal{D})}=\sup _{\substack{v \in C(\partial \mathcal{D}) \\ v \neq 0}} \frac{\left|\sum_{q \in \mathbb{Z}}\left(v, \zeta^{q}\right)_{L^{2}(\partial \mathcal{D})} d_{q}\right|}{\|v\|_{C(\partial \mathcal{D})}}=\|d\|_{-} .
$$

It remains to show that any continuous linear functional $f$ on $C(\partial \mathcal{D})$ has the form $f(v)=\left\langle v, d_{f}\right\rangle$ for some $d_{f} \in \mathfrak{C}(\partial \mathcal{D})$. According to the Riesz theorem, for any $f \in C^{\prime}(\partial \mathcal{D})$ there is a measure $d \mu$ supported on $\partial \mathcal{D}$ such that

$$
f(v)=\int_{\partial \mathcal{D}} v(\zeta) d \mu(\zeta) \text { for all } v \in C(\partial \mathcal{D}) .
$$

As we have noted above, for each $v \in C(\partial \mathcal{D})$ we have

$$
v=\sum_{q \in \mathbb{Z}}\left(v, \zeta^{q}\right)_{L^{2}(\partial \mathcal{D})} \zeta^{q},
$$

where the Fourier series converges uniformly on $\partial \mathcal{D}$. Hence

$$
f(v)=\sum_{q \in \mathbb{Z}}\left(v, \zeta^{q}\right)_{L^{2}(\partial \mathcal{D})} d_{q}
$$

where

$$
d_{q}=\int_{\partial \mathcal{D}} \zeta^{q} d \mu(\zeta)
$$

Finally, since the functional $f$ is continuous,

$$
\|f\|_{C^{\prime}(\partial \mathcal{D})}=\sup _{\substack{v \in C(\partial \mathcal{D}) \\ v \neq 0}} \frac{\left|\sum_{q \in \mathbb{Z}}\left(v, \zeta^{q}\right)_{L^{2}(\partial \mathcal{D})} d_{q}\right|}{\|v\|_{C(\partial \mathcal{D})}}=\|d\|_{-} ;
$$

in particular, the norm $\|d\|_{-}$is finite.

Thus we arrive at the following statement (cf. (3.12) above).

Lemma 4.2. Any eigenfunction of Problem 2.1 in the disk $\mathcal{D}$, corresponding to an eigenvalue $\lambda$, has the form

$$
u(z)=\sum_{q \in \mathbb{Z}}(z /|z|)^{q} \mathcal{J}_{q}(|z| \sqrt{\lambda}) d_{q}(\lambda)
$$

with some coefficients $\left\{d_{q}(\lambda)\right\}_{q \in \mathbb{Z}}$, satisfying $\|d(\lambda)\|_{-}<\infty$. Moreover, if the (relative) interiors of the sets $S$ and $\partial \mathcal{D} \backslash S$ are not empty, then the number of non-zero coefficients $d_{q}(\lambda)$ in the sum can not be finite. 
Proof. It follows from (4.5) that

$$
\begin{gathered}
\int_{\partial \mathcal{D}} \exp [\sqrt{-\lambda}(z \bar{\zeta}+\bar{z} \zeta) / 2] \zeta^{q} \frac{d \zeta}{2 \pi i \zeta}= \\
\int_{|\zeta=1|} \sum_{m=0}^{\infty}\left(\frac{|z| \sqrt{-\lambda}}{2}\right)^{m} \sum_{j=0}^{m} \frac{(z /|z|)^{2 j-m} \zeta^{m-2 j+q}}{j !(m-j) !} \frac{d \zeta}{2 \pi i \zeta}= \\
\sum_{\substack{(m+q) / 2 \in \mathbb{Z} \\
0 \leq(m+q) / 2 \leq m}}\left(\frac{|z| \sqrt{-\lambda}}{2}\right)^{m} \frac{(z /|z|)^{q}}{\left(\frac{m+q}{2}\right) !\left(\frac{m-q}{2}\right) !} .
\end{gathered}
$$

If $q \geq 0$ then $m \geq q$ and we substitute $(m-q) / 2=k$ :

$$
\begin{gathered}
\sum_{\substack{(m+q) / 2 \in \mathbb{Z} \\
0 \leq(m+q) / 2 \leq m}}\left(|z| \frac{\sqrt{-\lambda}}{2}\right)^{m} \frac{1}{\left(\frac{m+q}{2}\right) !\left(\frac{m-q}{2}\right) !}=\sum_{k \geq 0}\left(\frac{|z| \sqrt{-\lambda}}{2}\right)^{2 k+q} \frac{1}{k !(k+q) !}= \\
(\sqrt{-1})^{q} \mathcal{J}_{q}(|z| \sqrt{\lambda}) .
\end{gathered}
$$

If $q<0$ then $m \geq-q$ and we substitute $(m+q) / 2=k$ :

$$
\begin{aligned}
& \sum_{\substack{(m+q) / 2 \in \mathbb{Z} \\
0 \leq(m+q) / 2 \leq m}}\left(|z| \frac{\sqrt{-\lambda}}{2}\right)^{m} \frac{1}{\left(\frac{m+q}{2}\right) !\left(\frac{m-q}{2}\right) !}=\sum_{k \geq 0}\left(\frac{|z| \sqrt{-\lambda}}{2}\right)^{2 k+|q|} \frac{1}{k !(k+|q|) !}= \\
& (\sqrt{-1})^{|q|} \mathcal{J}_{-q}(|z| \sqrt{\lambda})=(\sqrt{-1})^{-q}(-1)^{q} \mathcal{J}_{q}(|z| \sqrt{\lambda})=(\sqrt{-1})^{q} \mathcal{J}_{q}(|z| \sqrt{\lambda}) .
\end{aligned}
$$

Hence formula (4.14) from Lemma 4.2 holds.

In particular, if $|z|=1$, we obtain the Laurent decomposition for the holomorphic function $\exp \left[\sqrt{-\lambda}\left(\zeta^{-1}+\zeta\right) / 2\right]$ in $\mathbb{C} \backslash\{0\}$ :

$$
\exp \left[\sqrt{-\lambda}\left(\zeta^{-1}+\zeta\right) / 2\right]=\sum_{q \in \mathbb{Z}}(\sqrt{-1})^{q} \mathcal{J}_{q}(\sqrt{\lambda}) \zeta^{-q}
$$

(this equality also follows from [1,9.44 and 9.45]).

Finally, the statement on the finite set of non-zero coefficients $d_{q}$ follows from Proposition 3.3.

Now let us indicate at least two examples where the expressions (4.9) and (4.10) can be analysed with the use of Lemma 4.1.

Example 4.3. Let $S=\emptyset$, i.e. $\alpha=2 \pi$, cf. Proposition 3.1. Then (4.2) is fulfilled automatically and we need to check (4.3) only. On the other hand, for all $m, j, p \in \mathbb{Z}_{+}, 2 j-p-m \neq 0$ we have

$$
\mathcal{K}_{m, j}^{(2)}(p, 2 \pi)=\frac{\exp [i 2 \pi(2 j-p-m)]-1}{2 j-p-m}=0 .
$$

Then (4.3) is true for all $p \in \mathbb{Z}$ if and only if for all $p \in \mathbb{Z}$ we have

$$
0=b_{0} \int_{|\zeta|=1} \sum_{\substack{0 \leq m+p \leq 2 m \\ \frac{m+p}{2} \in \mathbb{Z}_{+}}}\left(\frac{\sqrt{-\lambda}}{2}\right)^{m} \frac{2 \pi i \zeta^{-p} d \mu(\zeta)}{\left(\frac{m-p}{2}\right) !\left(\frac{m+p}{2}\right) !}+
$$




$$
\begin{gathered}
b_{1} \int_{|\zeta|=1} \sum_{\substack{0 \leq m-1+p \leq 2 m \\
\frac{m-1+p}{2} \in \mathbb{Z}_{+}}}\left(\frac{\sqrt{-\lambda}}{2}\right)^{m+1} \frac{2 \pi i \zeta^{-p} d \mu(\zeta)}{\left(\frac{m-1+p}{2}\right) !\left(\frac{m+1-p}{2}\right) !}+ \\
b_{2} \int_{|\zeta|=1} \sum_{\substack{0 \leq m+1+p \leq 2 m \\
\frac{m+1+p}{2} \in \mathbb{Z}_{+}}}\left(\frac{\sqrt{-\lambda}}{2}\right)^{m+1} \frac{2 \pi i \zeta^{-p} d \mu(\zeta)}{\left(\frac{m+1+p}{2}\right) !\left(\frac{m-1-p}{2}\right) !} .
\end{gathered}
$$

Substituting the summation indexes $((m+p) / 2=k$ in the first sum, $(m-$ $1+p) / 2=k$ in the second sum and $(m+1+p) / 2=k$ in the third sum), we see that this is equivalent to the following: for all $p \in \mathbb{Z}$

$$
\begin{gathered}
0=b_{0} \int_{|\zeta|=1} \sum_{k=(|p|+p) / 2}^{\infty}\left(\frac{\sqrt{-\lambda}}{2}\right)^{2 k-p} \frac{\zeta^{-p} d \mu(\zeta)}{k !(k-p) !}+ \\
b_{1} \int_{|\zeta|=1}^{\infty} \sum_{k=(|p-1|+p-1) / 2}^{\infty}\left(\frac{\sqrt{-\lambda}}{2}\right)^{2 k-p+2} \frac{\zeta^{-p} d \mu(\zeta)}{k !(k-p+1) !}+ \\
b_{2} \int_{|\zeta|=1}^{\infty} \sum_{k=(|p+1|+p+1) / 2}^{\infty}\left(\frac{\sqrt{-\lambda}}{2}\right)^{2 k-p} \frac{\zeta^{-p} d \mu(\zeta)}{k !(k-p-1) !}
\end{gathered}
$$

If $p<0$ then

$$
\begin{gathered}
\sum_{k=(|p|+p) / 2}^{\infty}\left(\frac{\sqrt{-\lambda}}{2}\right)^{2 k-p} \frac{1}{k !(k-p) !}=\sum_{k=0}^{\infty}\left(\frac{\sqrt{-\lambda}}{2}\right)^{2 k-p} \frac{1}{k !(k-p) !}= \\
(\sqrt{-1})^{|p|} \mathcal{J}_{|p|}(\sqrt{\lambda})=(\sqrt{-1})^{|p|}(-1)^{p} \mathcal{J}_{p}(\sqrt{\lambda})=(\sqrt{-1})^{p} \mathcal{J}_{p}(\sqrt{\lambda}) .
\end{gathered}
$$

If $p \geq 0$ then after re-indexation $n=k-p$ we obtain

$$
\begin{gathered}
\sum_{k=(|p|+p) / 2}^{\infty}\left(\frac{\sqrt{-\lambda}}{2}\right)^{2 k-p} \frac{1}{k !(k-p) !}= \\
\sum_{n=0}^{\infty}\left(\frac{\sqrt{-\lambda}}{2}\right)^{2 n+p} \frac{1}{n !(n+p) !}=(\sqrt{-1})^{p} \mathcal{J}_{p}(\sqrt{\lambda}) .
\end{gathered}
$$

Similarly,

$$
\begin{gathered}
\sum_{k=(|p-1|+p-1) / 2}^{\infty}\left(\frac{\sqrt{-\lambda}}{2}\right)^{2 k-(p-1)+1} \frac{1}{k !(k-p+1) !}=(\sqrt{-1})^{p} \sqrt{\lambda} \mathcal{J}_{p-1}(\sqrt{\lambda}) / 2, \\
\sum_{k=(|p+1|+p+1) / 2}^{\infty}\left(\frac{\sqrt{-\lambda}}{2}\right)^{2 k-(p+1)+1} \frac{\zeta^{-p} d \mu(\zeta)}{k !(k-p-1) !}=(\sqrt{-1})^{p} \sqrt{\lambda} \mathcal{J}_{p+1}(\sqrt{\lambda}) / 2 .
\end{gathered}
$$

Since $b_{1}+b_{2}=2$, using (4.16)-(4.20) and the famous relation (see, for instance, [1, 9.1.27])

$$
\mathcal{J}_{p+1}(r)+\mathcal{J}_{p-1}(r)=2 p \mathcal{J}_{p}(r) / r
$$


we see that (4.3) is true for all $p \in \mathbb{Z}$ if and only if for all $p \in \mathbb{Z}$ we have

$$
\left[\left(b_{0}-p b_{2}\right) \mathcal{J}_{p}(\sqrt{\lambda})+\sqrt{\lambda} \mathcal{J}_{p-1}(\sqrt{\lambda})\right] \int_{|\zeta|=1} \zeta^{-p} d \mu(\zeta)=0 .
$$

Now using Lemma 4.1 and (4.8) we conclude that for a measure $d \mu \in$ $\mathfrak{C}(\partial \mathcal{D})$ equality (4.3) is true for all $p \in \mathbb{Z}$ if and only if for all $p \in \mathbb{Z}$ we have

$$
\left[\left(b_{0}-p b_{2}\right) \mathcal{J}_{p}(\sqrt{\lambda})+\sqrt{\lambda} \mathcal{J}_{p-1}(\sqrt{\lambda})\right] d_{p}=0 .
$$

Clearly, the infinite system of linear equations (4.23) has a non-zero solution $d \in \mathfrak{C}(\partial D)$ if and only if

$$
G_{q}(\lambda)=\left(b_{0}-q b_{2}\right) \mathcal{J}_{p}(\sqrt{\lambda})+\sqrt{\lambda} \mathcal{J}_{p-1}(\sqrt{\lambda})=0
$$

for some $q \in \mathbb{Z}$. In this case as a solution we may take the measure

$$
d \mu_{q}(\zeta)=\frac{\zeta^{q}}{2 \pi(i)^{p+1}} \frac{d \zeta}{\zeta}, q \in \mathbb{Z}
$$

and the corresponding eigenvalues are the roots of the function $G_{q}(\lambda)$. In particular, the corresponding eigenfunctions are given as follows

$$
\begin{gathered}
u_{q}(z)=\int_{|\zeta|=1} \exp [\sqrt{-\lambda}(z \bar{\zeta}+\bar{z} \zeta) / 2] \frac{\zeta^{q}}{2 \pi(i)^{q+1}} \frac{d \zeta}{\zeta}= \\
\int_{|\zeta|=1} \sum_{m=0}^{\infty}\left(\frac{\sqrt{-\lambda}}{2}\right)^{m} \sum_{j=0}^{m} \frac{z^{j} \bar{z}^{m-j} \zeta^{m-j+q} \bar{\zeta}^{j}}{j !(m-j) ! 2 \pi(i)^{q+1}} \frac{d \zeta}{\zeta}= \\
\sum_{m=0}^{\infty}\left(\frac{\sqrt{-\lambda}}{2}\right)^{m} \frac{z^{(m+q) / 2} \bar{z}^{(m-q) / 2}}{(i)^{q}\left(\frac{m+q}{2}\right) !\left(\frac{m-q}{2}\right) !}=(z /|z|)^{q} \mathcal{J}_{q}(|z| \sqrt{\lambda}) .
\end{gathered}
$$

Thus we arrive at the results identical to that of Proposition 3.1.

Lemma 4.4. Let $S$ be the lower semicircle and $b_{0}+\left(b_{1} \cdot b_{2}\right)^{2} \neq 0$. Then

1. formula (4.2) is true for all $p \in \mathbb{Z}$ if and only if for all $p \in \mathbb{Z}$ we have

$$
\int_{|\zeta|=1} \zeta^{-2 p}\left(\pi(-1)^{p} \mathcal{J}_{2 p}(\sqrt{\lambda})-2 F_{p}^{(1)}(\zeta, \lambda)\right) d \mu(\zeta)=0
$$

where

$$
F_{p}^{(1)}(\zeta, \lambda)=\frac{\sqrt{\lambda}}{2} \sum_{k=0}^{\infty}\left(\frac{\sqrt{-\lambda}}{2}\right)^{2 k} \sum_{j=0}^{2 k+1} \frac{\zeta^{2 k+1-2 j+2 p}}{(2 k+1-2 j+2 p) j !(2 k+1-j) !}
$$

is a primitive for the holomorphic in $\mathbb{C} \backslash\{0\})$ function

$$
f_{p}^{(1)}(\zeta, \lambda)=\zeta^{2 p-1} \sin (\sqrt{\lambda}(\zeta+1 / \zeta) / 2)
$$

2. formula (4.3) is true for all $p \in \mathbb{Z}$ if and only if for all $p \in \mathbb{Z}$ we have

$$
\begin{gathered}
\int_{|\zeta|=1} \zeta^{-2 p}\left(\pi(-1)^{p}\left[\left(2\left(b_{0}-p b_{2}\right) \mathcal{J}_{2 p}(\sqrt{\lambda})+\sqrt{\lambda} \mathcal{J}_{2 p-1}(\sqrt{\lambda})\right]\right) d \mu(\zeta)+\right. \\
\sqrt{\lambda} \int_{|\zeta|=1} \zeta^{-2 p}\left(b_{1} F_{p-1}^{(2)}(\zeta, \lambda)+b_{2} F_{p}^{(2)}(\zeta, \lambda)\right) d \mu(\zeta)=0
\end{gathered}
$$


where

$$
F_{p}^{(2)}(\zeta, \lambda)=\sum_{k=0}^{\infty}\left(\frac{\sqrt{-\lambda}}{2}\right)^{2 k} \sum_{j=0}^{2 k} \frac{\zeta^{2 k+1-2 j+2 p}}{(2 k+1-2 j+2 p) j !(2 k-j) !}
$$

is a primitive for the holomorphic in $\mathbb{C} \backslash\{0\})$ function

$$
f_{p}^{(2)}(\zeta, \lambda)=\zeta^{2 p} \sqrt{\lambda} \cos (\sqrt{\lambda}(\zeta+1 / \zeta) / 2) .
$$

Proof. If $S$ is the lower semicircle then $\alpha=\pi$. Then $\lambda>0$,

$$
\frac{m}{2}+\frac{p \pi}{2 \pi-\alpha}=\frac{m}{2}+p
$$

and

$$
\begin{gathered}
\mathcal{K}_{m, j}^{(1)}(p, \pi)=\frac{\exp [i 2 \pi(2 j-2 p-m)]-\exp [i \pi(2 j-2 p \pi-m)]}{2 j-2 p-m}= \\
\begin{cases}0, & \text { if } m \text { is even, } \\
\frac{2}{2 j-2 p-m} & \text { if } m \text { is odd. }\end{cases}
\end{gathered}
$$

Hence, arguing as in Example 4.3 we see that (4.2) is true for all $p \in \mathbb{Z}$ if and only if for all $p \in \mathbb{Z}$ we have

$$
\begin{gathered}
0=\sum_{k=0}^{\infty}\left(\frac{\sqrt{-\lambda}}{2}\right)^{2 k-2 p} \frac{\pi i}{k !(k-2 p) !} \int_{|\zeta|=1} \zeta^{-2 p} d \mu(\zeta)- \\
2 \int_{|\zeta|=1} \sum_{k=0}^{\infty}\left(\frac{\sqrt{-\lambda}}{2}\right)^{2 k+1} \sum_{j=0}^{2 k+1} \frac{1}{2 k+1-2 j+2 p} \frac{\zeta^{2 k+1-2 j} d \mu(\zeta)}{j !(2 k+1-j) !}= \\
i\left[(-1)^{p} \pi \mathcal{J}_{2 p}(\lambda) \int_{|\zeta|=1} \zeta^{-2 p} d \mu(\zeta)-\right. \\
\left.\int_{|\zeta|=1} \sum_{k=0}^{\infty}\left(\frac{\sqrt{-\lambda}}{2}\right)^{2 k} \sum_{j=0}^{2 k+1} \frac{\sqrt{\lambda}}{2 k+1-2 j+2 p} \frac{\zeta^{2 k+1-2 j} d \mu(\zeta)}{j !(2 k+1-j) !}\right]
\end{gathered}
$$

It follows from (4.28) that (4.2) is true for all $p \in \mathbb{Z}$ if and only if for all $p \in \mathbb{Z}$ we have (4.24). Now consider the function

$$
\sin (\sqrt{\lambda}(\zeta+1 / \zeta) / 2)=\sum_{k=0}^{\infty} \frac{(-1)^{k}[\sqrt{\lambda}(\zeta+1 / \zeta) / 2]^{2 k+1}}{(2 k+1) !}
$$

holomorphic everywhere except the origin. Using the binomial formula one easily obtains that

$$
\zeta^{2 p-1} \sin (\sqrt{\lambda}(\zeta+1 / \zeta) / 2)=\frac{\sqrt{\lambda}}{2} \sum_{k=0}^{\infty}\left(\frac{\sqrt{-\lambda}}{2}\right)^{2 k} \sum_{j=0}^{2 k+1} \frac{\zeta^{2 k-2 j+2 p}}{j !(2 k+1-j) !}
$$

is holomorphic everywhere except the origin, too. Since $(2 k-2 j+2 p)$ never equals to $(-1)$, we see that the integral

$$
\int_{\gamma} \zeta^{2 p-1} \sin (\sqrt{\lambda}(\zeta+1 / \zeta) / 2) d \zeta=0
$$


for any simple closed curve $\gamma \subset \mathbb{C} \backslash\{0\}$. Hence (see, for instance, [21, Ch $2, \S 9$, corollary 3$]$ the function $f_{p}^{(1)}(\zeta, \lambda)$, given by $(4.25)$, admits a primitive $F_{p}^{(1)}(\zeta, \lambda)$ in $\mathbb{C} \backslash\{0\}$.

On the other hand,

$$
\begin{gathered}
\mathcal{K}_{m, j}^{(2)}(p, \pi)=\frac{\exp [i \pi(2 j-2 p-m)]-1}{2 j-2 p-m}= \\
\begin{cases}0, & \text { if } m \text { is even, } \\
\frac{2}{m-2 j+2 p} & \text { if } m \text { is odd. }\end{cases}
\end{gathered}
$$

Hence (4.3) is true for all $p \in \mathbb{Z}$ if and only if for all $p \in \mathbb{Z}$ we have

$$
\begin{aligned}
& 0=b_{0} \sum_{\substack{m \\
\frac{m}{2}+p \in \mathbb{Z}_{+} \\
0 \leq \frac{m}{2}+p \leq m}} \frac{\pi i}{\left(\frac{m}{2}-p\right) !\left(\frac{m}{2}+p\right) !}\left(\frac{\sqrt{-\lambda}}{2}\right)^{m} \int_{|\zeta|=1} \zeta^{-2 p} d \mu(\zeta)+ \\
& b_{0} \int_{|\zeta|=1} \sum_{m=0}^{\infty}\left(\frac{\sqrt{-\lambda}}{2}\right)^{m} \sum_{\substack{0 \leq j \leq m \\
m / 2 \notin \mathbb{Z}}} \frac{2}{m-2 j+2 p} \frac{\zeta^{m-2 j} d \mu(\zeta)}{j !(m-j) !}+ \\
& b_{1} \int_{|\zeta|=1} \sum_{\substack{\frac{m-1}{2}+p \in \mathbb{Z}_{+} \\
0 \leq \frac{m-1}{2}+p \leq m}}\left(\frac{\sqrt{-\lambda}}{2}\right)^{m+1} \frac{\pi i \zeta^{-2 p} d \mu(\zeta)}{\left(\frac{m}{2}-\frac{1}{2}+p\right) !\left(\frac{m}{2}+\frac{1}{2}-p\right) !}+ \\
& b_{1} \int_{|\zeta|=1} \sum_{m=0}^{\infty}\left(\frac{\sqrt{-\lambda}}{2}\right)^{m+1} \sum_{\substack{0 \leq j \leq m \\
m / 2 \in \mathbb{Z}}} \frac{2}{m-1-2 j+2 p} \frac{\bar{\zeta}^{j+1} \zeta^{m-j} d \mu(\zeta)}{j !(m-j) !}+ \\
& b_{2} \int_{|\zeta|=1} \sum_{\substack{\frac{m+1}{2}+p \in \mathbb{Z}_{+} \\
0 \leq \frac{m+1}{2}+p \leq m}}\left(\frac{\sqrt{-\lambda}}{2}\right)^{m+1} \frac{\pi i \zeta^{-2 p} d \mu(\zeta)}{\left(\frac{m+1}{2}+p\right) !\left(\frac{m-1}{2}-p\right) !}+ \\
& b_{2} \int_{|\zeta|=1} \sum_{m=0}^{\infty}\left(\frac{\sqrt{-\lambda}}{2}\right)^{m+1} \sum_{\substack{0 \leq j \leq m \\
m / 2 \in \mathbb{Z}}} \frac{2}{m+1-2 j+2 p} \frac{\bar{\zeta}^{j} \zeta^{m-j+1} d \mu(\zeta)}{j !(m-j) !}
\end{aligned}
$$

Using results of Example 4.3 and the calculations we have already done in the first part of the proof, we see that (4.29) reduces to the following: for all $p \in \mathbb{Z}$ we have

$$
\begin{gathered}
\int_{|\zeta|=1} \zeta^{-2 p}\left(\pi(-1)^{p}\left[\left(b_{0}-2 p b_{2}\right) \mathcal{J}_{2 p}(\sqrt{\lambda})+\sqrt{\lambda} \mathcal{J}_{2 p-1}(\sqrt{\lambda})\right]+2 b_{0} F_{p}^{(1)}(\zeta, \lambda)\right) d \mu(\zeta)+ \\
b_{1} \sqrt{\lambda} \sum_{k=0}^{\infty}\left(\frac{\sqrt{-\lambda}}{2}\right)^{2 k} \sum_{j=0}^{2 k} \frac{1}{2 k-1-2 j+2 p} \frac{\zeta^{2 k-2 j-1} d \mu(\zeta)}{j !(2 k-j) !}+ \\
b_{2} \sqrt{\lambda} \sum_{k=0}^{\infty}\left(\frac{\sqrt{-\lambda}}{2}\right)^{2 k} \sum_{j=0}^{2 k} \frac{1}{2 k+1-2 j+2 p} \frac{\zeta^{2 k-2 j+1} d \mu(\zeta)}{j !(2 k-j) !}=0
\end{gathered}
$$


Thus, using (4.30) we see that (4.3) is true for all $p \in \mathbb{Z}$ if and only if for all $p \in \mathbb{Z}$ we have

$$
\begin{gathered}
\int_{|\zeta|=1} \zeta^{-2 p}\left(\pi(-1)^{p}\left[\left(b_{0}-2 p b_{2}\right) \mathcal{J}_{2 p}(\sqrt{\lambda})+\sqrt{\lambda} \mathcal{J}_{2 p-1}(\sqrt{\lambda})\right]+2 b_{0} F_{p}^{(1)}(\zeta, \lambda)\right) d \mu(\zeta)+ \\
\sqrt{\lambda} \int_{|\zeta|=1} \zeta^{-2 p}\left(b_{1} F_{p-1}^{(2)}(\zeta, \lambda)+b_{2} F_{p}^{(2)}(\zeta, \lambda)\right) d \mu(\zeta)=0
\end{gathered}
$$

Taking into account (4.24), we finally conclude that (4.3) is true for all $p \in \mathbb{Z}$ if and only if for all $p \in \mathbb{Z}$ we have (4.26). It is not difficult to see that $F_{p}^{(2)}(\zeta, \lambda)$ is a primitive for the holomorphic function (4.27) in $\mathbb{C} \backslash\{0\}$.

Clearly, the integrands in formulas (4.24) and (4.26), i.e. the functions

$$
\begin{gathered}
\mathcal{F}_{p}^{(1)}(\zeta, \lambda)=\pi(-1)^{p} \mathcal{J}_{2 p}(\sqrt{\lambda})-2 F_{p}^{(1)}(\zeta, \lambda) \\
\mathcal{F}_{p}^{(2)}(\zeta, \lambda)=\pi(-1)^{p}\left[\left(2\left(b_{0}-p b_{2}\right) \mathcal{J}_{2 p}(\sqrt{\lambda})+\sqrt{\lambda} \mathcal{J}_{2 p-1}(\sqrt{\lambda})\right]+\right. \\
\sqrt{\lambda}\left(b_{1} F_{p-1}^{(2)}(\zeta, \lambda)+b_{2} F_{p}^{(2)}(\zeta, \lambda)\right)
\end{gathered}
$$

are primitives in $\mathbb{C} \backslash\{0\}$ for the holomorphic function $\left(-2 f_{p}^{(1)}(\zeta, \lambda)\right)$, given by (4.25) and for the holomorphic function

$$
\tilde{f}_{p}^{(2)}(\zeta, \lambda)=\zeta^{2 p-1} \sqrt{\lambda}\left(b_{2} \zeta+b_{1} \zeta^{-1}\right) \cos (\sqrt{\lambda}(\zeta+1 / \zeta) / 2)
$$

respectively. Now let us simplify (4.24) and (4.26) with the use of Lemmata 4.1, 4.4 and formula (4.8).

Lemma 4.5. The measure $d \mu=d \mu(\lambda) \in \mathfrak{C}(\partial \mathcal{D})$ satisfies (4.2) and (4.3) if and only if for any $p \in \mathbb{Z}$ we have

$$
\begin{gathered}
(-1)^{p} \pi \mathcal{J}_{2 p}(\sqrt{\lambda}) d_{2 p}(\lambda)+2 \sum_{q \in \mathbb{Z}} \frac{(-1)^{q} \mathcal{J}_{2 q-1}(\sqrt{\lambda})}{2 p+1-2 q} d_{2 q-1}(\lambda)=0 \\
\pi(-1)^{p}\left(2\left(b_{0}-p b_{2}\right) \mathcal{J}_{2 p}(\sqrt{\lambda})+\sqrt{\lambda} \mathcal{J}_{2 p-1}(\sqrt{\lambda})\right) d_{2 p}(\lambda)+ \\
\sum_{q \in \mathbb{Z}}\left(\frac{b_{1} \mathcal{J}_{2 q-2}(\sqrt{\lambda})}{2 p+1-2 q}-\frac{b_{2} \mathcal{J}_{2 q}(\sqrt{\lambda})}{2 p+1-2 q}\right)(-1)^{q-1} d_{2 q-1}(\lambda)=0
\end{gathered}
$$

Proof. Indeed, (4.8) yields

$$
\begin{gathered}
(2 \pi)^{-1}\left(\zeta^{-2 p} \mathcal{F}_{p}^{(1)}(\zeta, \lambda), \zeta^{-2 q}\right)_{L^{2}(\partial \mathcal{D})}= \\
\int_{|\zeta|=1} \zeta^{2 q-2 p}\left((-1)^{p} \pi \mathcal{J}_{2 p}(\sqrt{\lambda})-2 F_{p}^{(1)}(\zeta, \lambda)\right) \frac{d \zeta}{2 \pi i \zeta}= \\
(-1)^{p} \pi \mathcal{J}_{2 p}(\sqrt{\lambda}) \int_{|\zeta|=1} \zeta^{2 q-2 p} \frac{d \zeta}{2 \pi i \zeta}- \\
\int_{|\zeta|=1} \sum_{k=0}^{\infty}\left(\frac{\sqrt{-\lambda}}{2}\right)^{2 k} \sum_{j=0}^{2 k+1} \frac{\sqrt{\lambda} \zeta^{2 k+1-2 j+2 q} d \zeta}{2 \pi i \zeta(2 k+1-2 j+2 p) j !(2 k+1-j) !}=
\end{gathered}
$$




$$
\begin{cases}0, & p \neq q \\ (-1)^{p} \pi \mathcal{J}_{2 p}(\sqrt{\lambda}), & p=q .\end{cases}
$$

because $2 k+1-2 j+2 q \neq 0$. Similarly,

$$
\begin{gathered}
(2 \pi)^{-1}\left(\zeta^{-2 p} \mathcal{F}_{p}^{(2)}(\zeta, \lambda), \zeta^{-2 q}\right)_{L^{2}(\mathcal{} \mathcal{D})}= \\
\int_{|\zeta|=1} \zeta^{2 q-2 p}\left(\pi(-1)^{p}\left[\left(2\left(b_{0}-p b_{2}\right) \mathcal{J}_{2 p}(\sqrt{\lambda})+\sqrt{\lambda} \mathcal{J}_{2 p-1}(\sqrt{\lambda})\right]\right) \frac{d \zeta}{2 \pi i \zeta}+\right. \\
\sqrt{\lambda} \int_{|\zeta|=1} \zeta^{2 q-2 p}\left(b_{1} F_{p-1}^{(2)}(\zeta, \lambda)+b_{2} F_{p}^{(2)}(\zeta, \lambda)\right) \frac{d \zeta}{2 \pi i \zeta}= \\
\begin{cases}0, & p \neq q, \\
\pi(-1)^{p}\left[\left(2\left(b_{0}-p b_{2}\right) \mathcal{J}_{2 p}(\sqrt{\lambda})+\sqrt{\lambda} \mathcal{J}_{2 p-1}(\sqrt{\lambda})\right],\right. & p=q .\end{cases}
\end{gathered}
$$

Besides, using (4.8) we obtain

$$
\begin{gathered}
(2 \pi)^{-1}\left(\zeta^{-2 p} \mathcal{F}_{p}^{(1)}(\zeta, \lambda), \zeta^{1-2 q}\right)_{L^{2}(\partial \mathcal{D})}= \\
\int_{|\zeta|=1} \zeta^{2 q-1-2 p}\left((-1)^{p} \pi \mathcal{J}_{2 p}(\sqrt{\lambda})-2 F_{p}^{(1)}(\zeta, \lambda)\right) \frac{d \zeta}{2 \pi i \zeta}= \\
-\int_{|\zeta|=1} \sum_{k=0}^{\infty}\left(\frac{\sqrt{-\lambda}}{2}\right)^{2 k} \sum_{j=0}^{2 k+1} \frac{\sqrt{\lambda}}{2 k+1-2 j+2 p} \frac{\zeta^{2 k+1-2 j+2 q-1} d \zeta}{2 \pi i \zeta j !(2 k+1-j) !}= \\
-\sum_{\substack{k \geq 0 \\
0 \leq k+q \leq 2 k+1}}\left(\frac{\sqrt{-\lambda}}{2}\right)^{2 k} \frac{\sqrt{\lambda}}{2 p+1-2 q} \frac{1}{(k+q) !(k-q+1) !} .
\end{gathered}
$$

If $q \geq 1$ then $k \geq q-1$ and, substituting the indexes $m=k-q+1$, we obtain

$$
\begin{gathered}
\int_{|\zeta|=1} \zeta^{2 q-1-2 p}\left((-1)^{p} \pi \mathcal{J}_{2 p}(\sqrt{\lambda})-2 F_{p}^{(1)}(\zeta, \lambda)\right) \frac{d \zeta}{2 \pi i \zeta}= \\
\frac{-2}{2 p+1-2 q} \sum_{m=0}^{\infty}\left(\frac{\sqrt{\lambda}}{2}\right)^{2 m+2 q-1} \frac{(-1)^{m+q-1}}{(m+2 q-1) ! m !}=\frac{2(-1)^{q} \mathcal{J}_{2 q-1}(\sqrt{\lambda})}{2 p+1-2 q}
\end{gathered}
$$

If $q \leq 0$ then $k \geq-q$ and, substituting the indexes $m=k+q$, we obtain

In particular,

$$
\begin{gathered}
\int_{|\zeta|=1} \zeta^{2 q-1-2 p}\left((-1)^{p} \pi \mathcal{J}_{2 p}(\sqrt{\lambda})-2 F_{p}^{(1)}(\zeta, \lambda)\right) \frac{d \zeta}{2 \pi i \zeta}= \\
\frac{-2}{2 p+1-2 q} \sum_{m=0}^{\infty}\left(\frac{\sqrt{\lambda}}{2}\right)^{2 m-2 q+1} \frac{(-1)^{m-q}}{m !(m+1-2 q) !}= \\
\frac{2(-1)^{1-q} \mathcal{J}_{1-2 q}(\sqrt{\lambda})}{2 p+1-2 q}=\frac{2(-1)^{q} \mathcal{J}_{2 q-1}(\sqrt{\lambda})}{2 p+1-2 q} .
\end{gathered}
$$

$$
\zeta^{-2 p} F_{p}^{(1)}(\zeta, \lambda)=-\sum_{q \in \mathbb{Z}} \frac{(-1)^{q} \mathcal{J}_{2 q-1}(\sqrt{\lambda}) \zeta^{1-2 q}}{2 p+1-2 q} .
$$

Similarly,

$$
(2 \pi)^{-1}\left(\zeta^{-2 p} \mathcal{F}_{p}^{(2)}(\zeta, \lambda), \zeta^{1-2 q}\right)_{L^{2}(\partial \mathcal{D})}
$$




$$
\begin{gathered}
\int_{|\zeta|=1} \zeta^{2 q-2 p-1}\left(\pi(-1)^{p}\left[\left(2\left(b_{0}-p b_{2}\right) \mathcal{J}_{2 p}(\sqrt{\lambda})+\sqrt{\lambda} \mathcal{J}_{2 p-1}(\sqrt{\lambda})\right]\right) \frac{d \zeta}{2 \pi i \zeta}+\right. \\
\sqrt{\lambda} \int_{|\zeta|=1} \zeta^{2 q-2 p-1}\left(b_{1} F_{p-1}^{(2)}(\zeta, \lambda)+b_{2} F_{p}^{(2)}(\zeta, \lambda)\right) \frac{d \zeta}{2 \pi i \zeta}= \\
\sqrt{\lambda} \int_{|\zeta|=1} \zeta^{2 q-2 p-1}\left(b_{1} F_{p-1}^{(2)}(\zeta, \lambda)+b_{2} F_{p}^{(2)}(\zeta, \lambda)\right) \frac{d \zeta}{2 \pi i \zeta} \\
\int_{|\zeta|=1} \sum_{\substack{k=0 \\
\sum_{|\zeta|=1}}}\left(\frac{\sqrt{-\lambda}}{2}\right)^{2 k} \sum_{j=0}^{2 k-2 p-1} F_{p}^{(2)}(\zeta, \lambda) \frac{d \zeta}{2 \pi i \zeta}= \\
\sum_{\substack{k \geq 0 \\
0 \leq k+q \leq 2 k}}\left(\frac{\sqrt{-\lambda}}{2 k}\right)^{2 k} \frac{1}{2 p+1-2 q} \frac{1}{(k+q) !(k-q) !}
\end{gathered}
$$

If $q \leq 0$ then $k \geq-q$ and, substituting the indexes $m=k+q$, we obtain

$$
\begin{gathered}
\int_{|\zeta|=1} \zeta^{2 q-2 p-1} F_{p}^{(2)}(\zeta, \lambda) \frac{d \zeta}{2 \pi i \zeta}= \\
\frac{1}{2 p+1-2 q} \sum_{m=0}^{\infty}\left(\frac{\sqrt{-\lambda}}{2}\right)^{2 m-2 q} \frac{1}{m !(m-2 q) !} \\
=\frac{(-1)^{q} \mathcal{J}_{-2 q}(\sqrt{\lambda})}{(2 p+1-2 q)}=\frac{(-1)^{q} \mathcal{J}_{2 q}(\sqrt{\lambda})}{(2 p+1-2 q)} .
\end{gathered}
$$

If $q \geq 1$ then $k \geq q$, and substituting the indexes $m=k-q$, we obtain

$$
\begin{gathered}
\int_{|\zeta|=1} \zeta^{2 q-2 p-1} F_{p}^{(2)}(\zeta, \lambda) \frac{d \zeta}{2 \pi i \zeta}= \\
\frac{1}{2 p+1-2 q} \sum_{m=0}^{\infty}\left(\frac{\sqrt{-\lambda}}{2}\right)^{2 m+2 q} \frac{1}{(m+2 q) ! m !}=\frac{(-1)^{q} \mathcal{J}_{2 q}(\sqrt{\lambda})}{(2 p+1-2 q)}
\end{gathered}
$$

In particular,

$$
\zeta^{-2 p} F_{p}^{(2)}(\zeta, \lambda)=\sum_{q \in \mathbb{Z}} \frac{(-1)^{q} \mathcal{J}_{2 q}(\sqrt{\lambda}) \zeta^{1-2 q}}{2 p+1-2 q} .
$$

Again, by the same arguments, since the non-zero summand correspond to $2 j=2 k+2 q-2$,

$$
\begin{gathered}
\int_{|\zeta|=1} \zeta^{2 q-2 p-1} F_{p-1}^{(2)}(\zeta, \lambda) \frac{d \zeta}{2 \pi i \zeta}= \\
\int_{|\zeta|=1} \sum_{k=0}^{\infty}\left(\frac{\sqrt{-\lambda}}{2}\right)^{2 k} \sum_{j=0}^{2 k} \frac{1}{2 k-1-2 j+2 p} \frac{\zeta^{2 k-2 j-1+2 q-1} d \zeta}{2 \pi i \zeta j !(2 k-j) !}= \\
\sum_{\substack{k \geq 0 \\
0 \leq k+q-1 \leq 2 k}}^{\infty}\left(\frac{\sqrt{-\lambda}}{2}\right)^{2 k} \frac{1}{2 p+1-2 q} \frac{1}{(k+q-1) !(k-q+1) !}=
\end{gathered}
$$




$$
\frac{(-1)^{q-1} \mathcal{J}_{2 q-2}(\sqrt{\lambda})}{(2 p+1-2 q)}
$$

Finally, (4.2) and (4.3) follow from (4.24) and (4.26) and the form of the measure $d \mu(\lambda) \in \mathfrak{C}(\partial \mathcal{D})$.

It is clear now that the infinite system of linear equations (4.34), (4.35) is an analogue of the infinite system of linear equations (4.23) obtained in Example 4.3 for the case $S=\emptyset$.

Set now

$$
\gamma_{p, q}=\gamma_{p, q}(\lambda)=\alpha_{p}^{(1)}(\lambda) \beta_{p, q}^{(2)}(\lambda)-\alpha_{p}^{(2)}(\lambda) \beta_{p, q}^{(1)}(\lambda),
$$

where

$$
\begin{gathered}
\alpha_{p}^{(1)}=\alpha_{p}^{(1)}(\lambda)=\pi(-1)^{p} \mathcal{J}_{2 p}(\sqrt{\lambda}), \\
\alpha_{p}^{(2)}=\alpha_{p}^{(2)}(\lambda)=\pi(-1)^{p}\left(2\left(b_{0}-p b_{2}\right) \mathcal{J}_{2 p}(\sqrt{\lambda})+\sqrt{\lambda} \mathcal{J}_{2 p-1}(\sqrt{\lambda})\right), \\
\beta_{p, q}^{(1)}=\beta_{p, q}^{(1)}(\lambda)=\frac{2(-1)^{q} \mathcal{J}_{2 q-1}(\sqrt{\lambda})}{2 p+1-2 q}=\frac{\beta_{q, q}^{(1)}(\lambda)}{2 p+1-2 q}, \\
\beta_{p, q}^{(2)}=\beta_{p, q}^{(2)}(\lambda)=\left(b_{2} \mathcal{J}_{2 q}(\sqrt{\lambda})-b_{1} \mathcal{J}_{2 q-2}(\sqrt{\lambda})\right) \frac{(-1)^{q}}{2 p+1-2 q}=\frac{\beta_{q, q}^{(2)}(\lambda)}{2 p+1-2 q} .
\end{gathered}
$$

We fix the order of the coefficients $d_{q}, q \in \mathbb{Z}$, as follows:

$$
D^{T}=\left(d_{-1}, d_{0}, d_{1} d_{2} d_{-3}, d_{-2} \ldots d_{2 p-1}, d_{2 p}, d_{-2 p-1}, d_{-2 p} \ldots\right), p \in \mathbb{N} .
$$

Then (4.34) and (4.35) correspond to the infinite system of equations

$$
A(\lambda) D=0
$$

with an infinite "matrix" $A(\lambda)$ :

$$
\left(\begin{array}{cccccccc}
\beta_{0,0}^{(1)}(\lambda) & \alpha_{0}^{(1)}(\lambda) & \beta_{0,1}^{(1)}(\lambda) & 0 & \beta_{0,-1}^{(1)}(\lambda) & 0 & \beta_{0,2}^{(1)}(\lambda) & \ldots \\
\beta_{0,0}^{(2)}(\lambda) & \alpha_{0}^{(2)}(\lambda) & \beta_{0,1}^{(2)}(\lambda) & 0 & \beta_{0,-1}^{(2)}(\lambda) & 0 & \beta_{0,2}^{(2)}(\lambda) & \ldots \\
\beta_{1,0}^{(1)}(\lambda) & 0 & \beta_{1,1}^{(1)}(\lambda) & \alpha_{1}^{(1)}(\lambda) & \beta_{1,-1}^{(1)}(\lambda) & 0 & \beta_{1,2}^{(1)}(\lambda) & \ldots \\
\beta_{1,0}^{(2)}(\lambda) & 0 & \beta_{1,1}^{(2)}(\lambda) & \alpha_{1}^{(2)}(\lambda) & \beta_{1,-1}^{(2)}(\lambda) & 0 & \beta_{1,2}^{(2)}(\lambda) & \ldots \\
\beta_{-1,0}^{(1)}(\lambda) & 0 & \beta_{-1,1}^{(1)}(\lambda) & 0 & \beta_{-1,-1}^{(1)}(\lambda) & \alpha_{-1}^{(1)}(\lambda) & \beta_{-1,2}^{(1)}(\lambda) & \ldots \\
\beta_{-1,0}^{(2)}(\lambda) & 0 & \beta_{-1,1}^{(2)}(\lambda) & 0 & \beta_{-1,-1}^{(2)}(\lambda) & \alpha_{-1}^{(2)}(\lambda) & \beta_{-1,2}^{(2)}(\lambda) & \ldots \\
\beta_{2,0}^{(1)}(\lambda) & 0 & \beta_{2,1}^{(1)}(\lambda) & 0 & \beta_{2,-1}^{(1)}(\lambda) & 0 & \beta_{2,2}^{(1)}(\lambda) & \ldots \\
\beta_{2,0}^{(2)}(\lambda) & 0 & \beta_{2,1}^{(2)}(\lambda) & 0 & \beta_{2,-1}^{(2)}(\lambda) & 0 & \beta_{2,2}^{(2)}(\lambda) & \ldots \\
\ldots & \ldots & \ldots & \ldots & \ldots & \ldots & \ldots & \ldots \\
\ldots & \ldots & \ldots & \ldots & \ldots & \ldots & \ldots & \ldots
\end{array}\right)
$$

and the set of the coefficients $\left\{d_{q}\right\}$ having the finite norm $\|d\|_{-}$.

First, we note that for any fixed $p \in \mathbb{Z}$ the numbers $\alpha_{p}^{(1)}(\lambda)$ and $\alpha_{p}^{(2)}(\lambda)$ can not vanish simultaneously because of the Siegel theorem on common zeroes of the Bessel functions (see [24, pp. 484-485]). 
Then (meaning that for each $p \in \mathbb{Z}$ there is $i_{p}=1$ or $i_{p}=2$ such that $\alpha_{p}^{\left(i_{p}\right)}(\lambda) \neq 0$ ) we obtain an equivalent infinite system of linear equations

$$
\tilde{A}(\lambda) D=0
$$

where

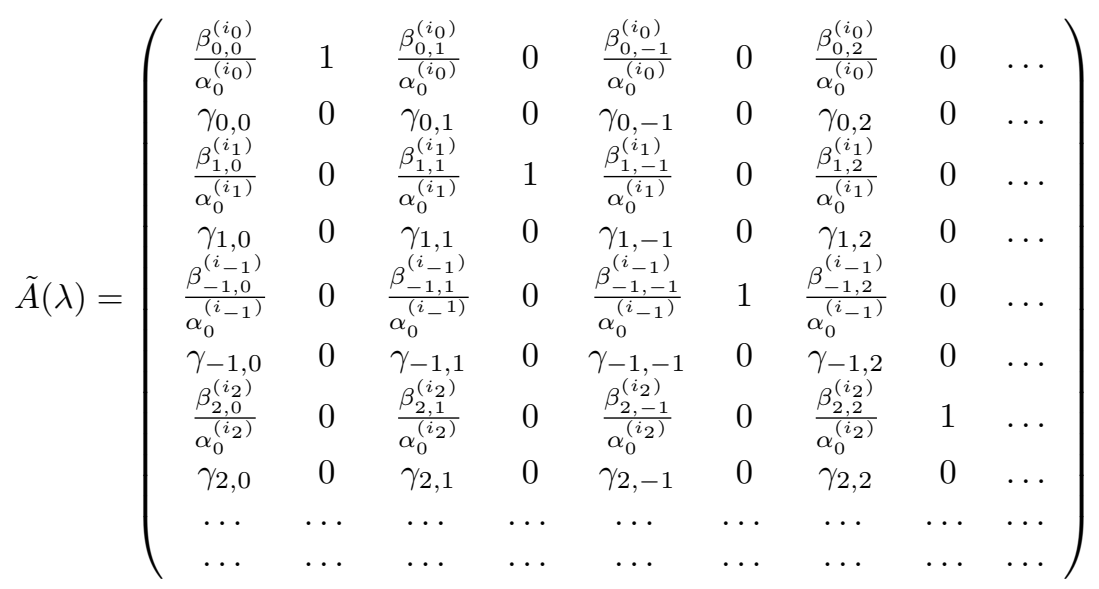

Thus we arrive at the following theorem.

Theorem 4.6. Let the (relative) interiors of the sets $S$ and $\partial \mathcal{D} \backslash S$ on $\partial \mathcal{D}$ be not empty. If $b_{0}^{2}+\left(b_{1} \cdot b_{2}\right)^{2}=0$ then a number $\lambda>0$ is an eigenvalue of Zaremba problem (3.2) with $a_{0}=0$ if and only if there is a non-zero vector $D=D(\lambda)$ with the coefficients $\left\{d_{q}(\lambda)\right\}$ having the finite norm $\|d(\lambda)\|_{-}$such that its non-zero odd part

$$
D_{\mathrm{o} d d}^{T}(\lambda)=\left(d_{-1}(\lambda), d_{1}(\lambda) d_{-3}(\lambda), \ldots d_{2 p-1}(\lambda), d_{-2 p-1}(\lambda), \ldots\right), p \in \mathbb{N},
$$

satisfies

$$
\tilde{A}_{\mathrm{o} d d}(\lambda) D_{\mathrm{o} d d}(\lambda)=0
$$

where

$$
\tilde{A}_{\mathrm{o} d d}(\lambda)=\left(\begin{array}{ccccccc}
\gamma_{0,0}(\lambda) & \gamma_{0,1}(\lambda) & \gamma_{0,-1}(\lambda) & \ldots & \gamma_{0, p}(\lambda) & \gamma_{0,-p}(\lambda) & \ldots \\
\gamma_{1,0}(\lambda) & \gamma_{1,1}(\lambda) & \gamma_{1,-1}(\lambda) & \ldots & \gamma_{1, p}(\lambda) & \gamma_{1,-p}(\lambda) & \ldots \\
\gamma_{-1,0}(\lambda) & \gamma_{-1,1}(\lambda) & \gamma_{-1,-1}(\lambda) & \ldots & \gamma_{-1, p}(\lambda) & \gamma_{-1,-p}(\lambda) & \ldots \\
\ldots & \ldots & \ldots & \ldots & \ldots & \ldots & \ldots \\
\gamma_{p, 0}(\lambda) & \gamma_{p, 1}(\lambda) & \gamma_{p,-1}(\lambda) & \ldots & \gamma_{p, p}(\lambda) & \gamma_{p,-p}(\lambda) & \ldots \\
\gamma_{-p, 0}(\lambda) & \gamma_{-p, 1}(\lambda) & \gamma_{-p,-1}(\lambda) & \ldots & \gamma_{-p, p}(\lambda) & \gamma_{-p,-p}(\lambda) & \ldots \\
\ldots & \ldots & \ldots & \ldots & \ldots & \ldots & \ldots
\end{array}\right)
$$

Besides the corresponding measure $d \mu(\lambda) \in \mathfrak{C}(\partial \mathcal{D})$ can not have finite number of the non-zero coefficients $d_{q}(\lambda)$.

On the other hand, as we have seen above, the Siegel theorem on the common zeroes of the Bessel functions sets some restrictions on the simultaneous vanishing of the determinants $\gamma_{p, q}(\lambda)$ 
A disadvantage of Theorem 4.6 is that we need to control the growth of the coefficients $\left\{d_{q}(\lambda)\right\}$. However we can improve the situation using HahnBanach Theorem.

As usual, we split any function $v \in C(\partial \mathcal{D})$ into the sum of the odd and even parts:

$$
v(\zeta)=v_{\text {odd }}(\zeta)+v_{\text {even }}(\zeta)
$$

where

$$
v_{\text {odd }}(\zeta)=[v(\zeta)-v(-\zeta)] / 2, v_{\text {even }}(\zeta)=[v(\zeta)+v(-\zeta)] / 2 .
$$

Since $v_{\text {odd }}, v_{\text {even }} \in C(\partial \mathcal{D})$, the corresponding Fourier series converge uniformly on $\partial \mathcal{D}$ :

$$
\begin{gathered}
v(\zeta)=\sum_{p \in \mathbb{Z}}\left(v, \zeta^{p}\right)_{L^{2}(\partial \mathcal{D})} \zeta^{p} \\
v_{\text {even }}(\zeta)=\sum_{p \in \mathbb{Z}}\left(v, \zeta^{2 p}\right)_{L^{2}(\partial \mathcal{D})} \zeta^{2 p}, v_{\text {odd }}(\zeta)=\sum_{p \in \mathbb{Z}}\left(v, \zeta^{2 p-1}\right)_{L^{2}(\partial \mathcal{D})} \zeta^{2 p-1} .
\end{gathered}
$$

In particular, the space $C(\partial \mathcal{D})$ splits on the direct sum

$$
C(\partial \mathcal{D})=C_{\text {odd }}(\partial \mathcal{D}) \oplus C_{\text {even }}(\partial \mathcal{D})
$$

with the continuous natural projectors.

Corollary 4.7. Let the (relative) interiors of the sets $S$ and $\partial \mathcal{D} \backslash S$ on $\partial \mathcal{D}$ be not empty. If $b_{0}^{2}+\left(b_{1} \cdot b_{2}\right)^{2}=0$ then the following conditions are equivalent:

1) a number $\lambda>0$ is an eigenvalue of Zaremba problem (3.2) with $a_{0}=0$;

2) the system $\left\{\zeta^{-2 p} \mathcal{F}_{p}^{(1)}(\zeta, \lambda), \zeta^{-2 p} \mathcal{F}_{p}^{(2)}(\zeta, \lambda)\right\}_{p \in \mathbb{Z}}$, where functions $\mathcal{F}_{p}^{(1)}(\zeta, \lambda)$ and $\mathcal{F}_{p}^{(2)}(\zeta, \lambda)$ are given by (4.32) and (4.33), respectively, is not complete in $C(\partial \mathcal{D})$;

3) the system

$$
\left\{\Phi_{p}(\zeta, \lambda)=\zeta^{-2 p}\left(\alpha_{p}^{(1)}(\lambda) \mathcal{F}_{p}^{(2)}(\zeta, \lambda)-\alpha_{p}^{(2)}(\lambda) \mathcal{F}_{p}^{(1)}(\zeta, \lambda)\right)\right\}_{p \in \mathbb{Z}}
$$

is not complete in $C_{\text {odd }}(\partial \mathcal{D})$.

Proof. The equivalence of conditions 1) and 2) of the corollary follows from Hahn-Banach Theorem and formulae (4.24), (4.26).

To prove the equivalence of conditions 2) and 3) we note that any measure $d \mu \in \mathfrak{C}(\partial \mathcal{D})$ splits naturally as

$$
d \mu=d \mu_{\text {even }}+d \mu_{\text {odd }}
$$

with

$$
\begin{aligned}
& d \mu_{\text {even }}=\sum_{p \in \mathbb{Z}} d_{2 p} \zeta^{2 p}, d \mu_{\text {odd }}=\sum_{p \in \mathbb{Z}} d_{2 p-1} \zeta^{2 p}, \\
& d \mu_{\text {odd }}=d \mu_{\mid C_{\text {odd }}(\partial \mathcal{D})}, d \mu_{\text {even }}=d \mu_{\mid C_{\text {even }}(\partial \mathcal{D})} .
\end{aligned}
$$

As we have seen in the proof of Lemma 4.5 , for each $p \in \mathbb{Z}$ there is $j_{p}=1$ or $j_{p}=2$ such $\alpha_{p}^{\left(j_{p}\right)}(\lambda) \neq 0$. Hence the linear span of the system 
$\left\{\zeta^{-2 p} \mathcal{F}_{p}^{(1)}(\zeta, \lambda), \zeta^{-2 p} \mathcal{F}_{p}^{(2)}(\zeta, \lambda)\right\}_{p \in \mathbb{Z}}$ coincides with the linear span of the system

$$
\left\{\zeta^{-2 p} \mathcal{F}_{p}^{\left(j_{p}\right)}(\zeta, \lambda) / \alpha_{p}^{\left(j_{p}\right)}(\lambda), \zeta^{-2 p}\left(\alpha_{p}^{(2)}(\lambda) \mathcal{F}_{p}^{(1)}(\zeta, \lambda)-\alpha_{p}^{(1)}(\lambda) \mathcal{F}_{p}^{(2)}(\zeta, \lambda)\right)\right\}_{p \in \mathbb{Z}}
$$

Now, following the calculations of Lemma 4.5, we see that

$$
\zeta^{-2 p} \mathcal{F}_{p}^{(j)}(\zeta, \lambda)=\zeta^{2 p} \alpha_{p}^{(j)}(\lambda)+\sum_{q \in \mathbb{Z}} \beta_{p, q}^{(j)}(\lambda) \zeta^{1-2 q}
$$

and therefore

$$
\Phi_{p}(\zeta, \lambda)=\zeta^{-2 p}\left(\alpha_{p}^{(1)}(\lambda) \mathcal{F}_{p}^{(2)}(\zeta, \lambda)-\alpha_{p}^{(2)}(\lambda) \mathcal{F}_{p}^{(1)}(\zeta, \lambda)\right)=\sum_{q \in \mathbb{Z}} \gamma_{p, q}(\lambda) \zeta^{1-2 q}
$$

By the very definition the elements of the system (4.42) belong to $C_{\text {odd }}(\partial \mathcal{D})$. Hence Theorem 4.6 means that the number $\lambda$ is an eigenvalue of Zaremba problem (3.2) with $a_{0}=0$; if and only if there is non-zero measure $d \mu \in \mathfrak{C}(\partial \mathcal{D})$ with the non-zero odd part $d \mu_{\text {odd }}$ such that

$$
\int_{\partial \mathcal{D}} \zeta^{-2 p}\left(\alpha_{p}^{(1)}(\lambda) \mathcal{F}_{p}^{(2)}(\zeta, \lambda)-\alpha_{p}^{(2)}(\lambda) \mathcal{F}_{p}^{(1)}(\zeta, \lambda)\right) d \mu_{\text {odd }}(\zeta)=0
$$

for all $p \in \mathbb{Z}$. Finally, applying Hahn-Banach Theorem we conclude that this statement is equivalent to the condition 3) of the corollary.

According to Theorem 4.6 a number $\lambda>0$ is an eigenvalue of Zaremba problem (3.2) with $a_{0}=0$ if and only if there is $d \in \mathfrak{C}(\partial D)$ such that

$$
\sum_{p \in \mathbb{Z}}\left(\sum_{q \in \mathbb{Z}} \gamma_{p, q}(\lambda) d_{2 q-1}\right) z^{1-2 p}=0 \text { for all } z \in \mathbb{C} \backslash\{0\} .
$$

On the other hand, the functions $\Phi_{p}(\zeta, \lambda)$ are holomorphic in $\mathbb{C} \backslash\{0\}$. Hence their Laurent series converge uniformly with respect to $\zeta$ on any compact in $\mathbb{C} \backslash\{0\}$. Thus, since the sequence $\left\{\gamma_{p, q}(\lambda)\right\}_{q \in \mathbb{Z}}$ consists of the Laurent coefficients of the function $\Phi_{p}(\zeta, \lambda)$ (see (4.43)), we may change the order in (4.44) and obtain the equivalence of (4.44) and the following equality

$$
\sum_{q \in \mathbb{Z}} d_{2 q-1}\left(\sum_{p \in \mathbb{Z}} \gamma_{p, q}(\lambda) z^{1-2 p}\right)=0 \text { for all } z \in \mathbb{C} \backslash\{0\}
$$

Since

$$
\begin{gathered}
\alpha_{p}^{(1)}(\lambda) \beta_{p, q}^{(2)}(\lambda)=\pi(-1)^{q}\left(b_{2} \mathcal{J}_{2 q}(\sqrt{\lambda})-b_{1} \mathcal{J}_{2 q-2}(\sqrt{\lambda})\right) \frac{(-1)^{p} \mathcal{J}_{2 p}(\sqrt{\lambda})}{2 p+1-2 q}= \\
-\pi(-1)^{q}\left(b_{2} \mathcal{J}_{2 q}(\sqrt{\lambda})-b_{1} \mathcal{J}_{2 q-2}(\sqrt{\lambda})\right) \frac{(-1)^{p} \mathcal{J}_{2 p}(\sqrt{\lambda})}{2(q-1)+1-2 p}
\end{gathered}
$$

formula (4.37) implies that

$$
\begin{gathered}
\sum_{p \in \mathbb{Z}} \alpha_{p}^{(1)}(\lambda) \beta_{p, q}^{(2)}(\lambda) z^{1-2 p}= \\
\pi(-1)^{q+1}\left(b_{2} \mathcal{J}_{2 q}(\sqrt{\lambda})-b_{1} \mathcal{J}_{2 q-2}(\sqrt{\lambda})\right) z^{-2(q-1)} F_{q-1}^{(2)}(z, \lambda)=
\end{gathered}
$$


Similarly, since

$$
-\pi \beta_{q, q}^{(2)} z^{-2(q-1)} F_{q-1}^{(2)}(z, \lambda)
$$

$$
\begin{gathered}
\frac{\alpha_{p}^{(2)}(\lambda) \beta_{p, q}^{(1)}(\lambda)}{2 \pi(-1)^{q} \mathcal{J}_{2 q-1}(\sqrt{\lambda})}=\frac{(-1)^{p}\left(\left(2\left(b_{0}-p b_{2}\right) \mathcal{J}_{2 p}(\sqrt{\lambda})+\sqrt{\lambda} \mathcal{J}_{2 p-1}(\sqrt{\lambda})\right)\right.}{2 p+1-2 q}= \\
-\frac{(-1)^{p}\left(\left(2 b_{0}-(2 q-1) b_{1}+(2(q-1)+1-2 p) b_{2} \mathcal{J}_{2 p}(\sqrt{\lambda})+\sqrt{\lambda} \mathcal{J}_{2 p-1}(\sqrt{\lambda})\right)\right.}{2(q-1)+1-2 p},
\end{gathered}
$$

formulae (4.36), (4.37) imply that

$$
\begin{aligned}
& \sum_{p \in \mathbb{Z}} \frac{\alpha_{p}^{(2)}(\lambda) \beta_{p, q}^{(1)}(\lambda) z^{1-2 p}}{2 \pi(-1)^{q} \mathcal{J}_{2 q-1}(\sqrt{\lambda})}=-b_{2} \sum_{p \in \mathbb{Z}}(-1)^{p} \mathcal{J}_{2 p}(\sqrt{\lambda}) z^{1-2 p}- \\
& z^{-2(q-1)}\left(\left(2 b_{0}-(2 q-1) b_{1}\right) F_{q-1}^{(2)}(z, \lambda)-\sqrt{\lambda} F_{q-1}^{(1)}(z, \lambda)\right) .
\end{aligned}
$$

Finally, we note that, according to (4.15) and the Euler formula,

$$
\sum_{p \in \mathbb{Z}}(-1)^{p} \mathcal{J}_{2 p}(\sqrt{\lambda}) z^{-2 p}=\cos \left[\sqrt{\lambda}\left(z+z^{-1}\right) / 2\right], z \neq 0 .
$$

and therefore we obtain a holomorphic function in $\mathbb{C} \backslash\{0\}$ :

$$
\begin{gathered}
\Psi_{q}(z, \lambda)=\sum_{p \in \mathbb{Z}} \gamma_{p, q}(\lambda) z^{1-2 p}=2 b_{2} \pi(-1)^{q} \mathcal{J}_{2 q-1}(\sqrt{\lambda}) z \cos \left[\sqrt{\lambda}\left(z+z^{-1}\right) / 2\right]+ \\
2 \pi(-1)^{q} \mathcal{J}_{2 q-1}(\sqrt{\lambda}) z^{-2(q-1)}\left(\left(2 b_{0}-(2 q-1) b_{1}\right) F_{q-1}^{(2)}(z, \lambda)-\sqrt{\lambda} F_{q-1}^{(1)}(z, \lambda)\right)+ \\
\pi(-1)^{q}\left(b_{1} \mathcal{J}_{2 q-2}(\sqrt{\lambda})-b_{2} \mathcal{J}_{2 q}(\sqrt{\lambda})\right) z^{-2(q-1)} F_{q-1}^{(2)}(z, \lambda)= \\
2 b_{2} \pi(-1)^{q} \mathcal{J}_{2 q-1}(\sqrt{\lambda}) z \cos \left[\sqrt{\lambda}\left(z+z^{-1}\right) / 2\right]+ \\
\pi(-1)^{q} z^{-2(q-1)}\left(-2 \sqrt{\lambda} \mathcal{J}_{2 q-1}(\sqrt{\lambda}) F_{q-1}^{(1)}(z, \lambda)+\right. \\
\left.\left(2 \mathcal{J}_{2 q-1}(\sqrt{\lambda})\left(2 b_{0}-(2 q-1) b_{1}\right)+b_{1} \mathcal{J}_{2 q-2}(\sqrt{\lambda})-b_{2} \mathcal{J}_{2 q}(\sqrt{\lambda})\right) F_{q-1}^{(2)}(z, \lambda)\right) .
\end{gathered}
$$

Hence we obtain the following.

Corollary 4.8. Let the (relative) interiors of the sets $S$ and $\partial \mathcal{D} \backslash S$ on $\partial \mathcal{D}$ be not empty. If $b_{0}^{2}+\left(b_{1} \cdot b_{2}\right)^{2}=0$ then a number $\lambda>0$ is an eigenvalue of Zaremba problem (3.2) with $a_{0}=0$ if and only if there is a non-zero set of the coefficients $\left\{d_{p}(\lambda)\right\}_{p \in \mathbb{Z}}$ such that $\|d(\lambda)\|_{-}<\infty$,

$$
\sum_{p \in \mathbb{Z}} d_{2 p-1}(\lambda) \Psi_{p}(z, \lambda)=0 \text { for all } z \in \partial \mathcal{D}
$$

for the analytic functions $\Psi_{p}(z, \lambda)$ in $\mathbb{C} \backslash\{0\}$, given by (4.46), and

$$
d_{2 p}(\lambda)=-\sum_{q \in \mathbb{Z}} d_{2 q-1} \frac{\beta_{p, q}^{\left(i_{p}\right)}(\lambda)}{\alpha_{p}^{\left(i_{p}\right)}(\lambda)}
$$




\section{Acknowledgment}

The work was supported by the grant of the Russian Federation Government for scientific research under the supervision of leading scientist at the Siberian Federal University, contract N. 14.Y26.31.0006, and by RFBR grant 14-0100544.

\section{References}

[1] M. Abramowitz, I. Stegun, Handbook of Mathematical Functions with formulas, graphs and mathematical tables. Washington, 1972.

[2] S. Agmon, On the eigenfunctions and on the eigenvalues of general elliptic boundary value problems, Comm. Pure Appl. Math. 15 (1962), 119-147.

[3] M. S. Agranovich, Spectral Problems in Lipschitz Domains, Uspekhi Mat. Nauk 57 (2002), No. 347 (5), 3-78.

[4] M. S. Agranovich, Spectral Problems in Lipschitz Domains, Modern Mathematics, Fundamental Trends 39 (2011), 11-35.

[5] F. E. Browder, On the eigenfunctions and eigenvalues of the general elliptic differential operator, Proc. Nat. Acad. Sci. USA 39 (1953), 433-439.

[6] F. E. BRowder, On the spectral theory of strongly elliptic differential operators, Proc. Nat. Acad. Sci. USA 45 (1959), 1423-1431.

[7] N. Dunford and Schwartz, J. T., Linear Operators, Vol. II, Selfadjoint Operators in Hilbert Space, Intersci. Publ., New York, 1963.

[8] Yu. Egorov, V. Kondratiev and B. W. Schulze, Completeness of eigenfunctions of an elliptic operator on a manifold with conical points, Russ. J. Math. Phys. 8 (2001), no. 3, 267-274.

[9] I. Ts. Gokhberg and M. G. Krein, Introduction to the Theory of Linear Nonselfadjoint Operators in Hilbert Spaces, AMS, Providence, R.I., 1969.

[10] M. V. Keldysh, On the characteristic values and characteristic functions of certain classes of non-selfadjoint equations, Dokl. AN SSSR 77 (1951), 11-14.

[11] J. J. Koнn, Subellipticity of the $\bar{\partial}$-Neumann problem on pseudoconvex domains: sufficient conditions, Acta Math. 142 (1979), No. 1-2, 79-122.

[12] V. A. Kondrat'Ev, Completeness of the systems of root functions of elliptic operators in Banach spaces, Russ. J. Math. Phys. 6 (1999), No. 10, 194-201.

[13] V. P. Mikhailov, Partial Differential Equations, Nauka, Moscow, 1976.

[14] C. B. Morrey and L. Nirenberg, On the analyticity of the solutions of linear elliptic systems of partial differential equations, Comm. Pure and Appl. Math. 10 (1957), 271-290.

[15] V. Palamodov, Linear differential operators with constant coefficients, Nauka, Moscow, 1967. English transl. in Springer-Verlag, Berlin-Heidelberg-New York, 1970.

[16] A. Polkovnikov, A. Shlapunov, On the spectral properties of a non-coercive mixed problem associated with $\bar{\partial}$-operator, J. Siberian Fed. Uni. 6 (2013), No. 2.

[17] M. Schechter, Negative norms and boundary problems, Ann. Math. 72 (1960), No. 3, 581-593. 
[18] A. Shlapunov, N. Tarkhanov, Bases with double orthogonality in the Cauchy problem for systems with injective symbols, Proc. London. Math. Soc., 71 (1995), N. 1, P. 1-54.

[19] A. Shlapunov, N. Tarkhanov, On completeness of root functions of SturmLiouville problems with discontinuous boundary operators, J. of Differential Equations, 10 (2013), 3305-3337.

[20] A. Shlapunov, N. Tarkhanov, Sturm-Liouville Problems in Weighted Spaces over Domains with Non-Smooth Edges. I, Mat. Trudy, V.18, N. 1 (2015), 118 189. English transl. in Siberian Advances in Mathematics, V. 26, No. 1 (2016), 30-76.

[21] Yu. Sidorov, M. Fedoryuk, M. Shabunin, Lectures on function's theory of complex variables. Nauka, Moscow, 1982.

[22] N. TARKhanov, On the root functions of general elliptic boundary value problems, Compl. Anal. Oper. Theory, 1 (2006), 115-141.

[23] A. N. Tikhonov, A. A. Samarskit, Equations of Mathematical Physics, Nauka, Moscow, 1972.

[24] G. N. Watson, A treatise on the theory of Bessel functions. Second Edition. Oxford Univrsity Press, London, 1944.

[25] S. Zaremba, Sur un problème mixte relatif à l'équation de Laplace, Bull. Acad. Sci. Cracovie (1910), 314-344.

Ari Laptev

Imperial College London

Huxley Building, 180 Queen's Gate

London SW7 2AZ

United Kingdom

Siberian Federal University

pr. Svobodnyi 79

660041 Krasnoyarsk

Russia

e-mail: E-mail address: a.laptev@imperial.ac.uk

Anastasiya Peicheva

Siberian Federal University

Institute of Mathematics and Computer Science

pr. Svobodnyi 79

660041 Krasnoyarsk

Russia

e-mail: peichevaas@mail.ru

Alexander Shlapunov

Siberian Federal University

Institute of Mathematics and Computer Science

pr. Svobodnyi 79

660041 Krasnoyarsk

Russia

e-mail: ashlapunov@sfu-kras.ru 